\title{
Propagation of statistical and nuclear data uncertainties in Monte Carlo burn-up calculations
}

\author{
Nuria García-Herranz ，Oscar Cabellos ，Javier Sanz ，Jesús Juan ，Jim C. Kuijper \\ Departamento de Ingeniería Nuclear, Universidad Politécnica de Madrid, UPM, Spain \\ Departamento de Ingeniería Energética, Universidad Nacional de Educación a Distancia, UNED, Spain \\ Laboratorio de Estadística, Universidad Politécnica de Madrid, UPM, Spain \\ NRG-Fuels, Actinides \& Isotopes Group, Petten, The Netherlands
}

\begin{abstract}
Two methodologies to propagate the uncertainties on the nuclide inventory in combined Monte Carlo-spectrum and burn-up calculations are presented, based on sensitivity/uncertainty and random sampling techniques (uncertainty Monte Carlo method). Both enable the assessment of the impact of uncertainties in the nuclear data as well as uncertainties due to the statistical nature of the Monte Carlo neutron transport calculation. The methodologies are implemented in our MCNP-ACAB system, which combines the neutron transport code MCNP-4C and the inventory code ACAB.

A high burn-up benchmark problem is used to test the MCNP-ACAB performance in inventory predictions, with no uncertainties. A good agreement is found with the results of other participants.

This benchmark problem is also used to assess the impact of nuclear data uncertainties and statistical flux errors in high burn-up applications. A detailed calculation is performed to evaluate the effect of cross-section uncertainties in the inventory prediction, taking into account the temporal evolution of the neutron flux level and spectrum. Very large uncertainties are found at the unusually high burn-up of this exercise $(800 \mathrm{MWd} / \mathrm{kgHM})$. To compare the impact of the statistical errors in the calculated flux with respect to the cross uncertainties, a simplified problem is considered, taking a constant neutron flux level and spectrum. It is shown that, provided that the flux statistical deviations in the Monte Carlo transport calculation do not exceed a given value, the effect of the flux errors in the calculated isotopic inventory are negligible (even at very high burn-up) compared to the effect of the large cross-section uncertainties available at present in the data files.
\end{abstract}

\section{Introduction}

Most of nuclear systems, from the present LWR's to the future designs, require a reliable isotopic inventory prediction for aspects related to operation, safety and waste management purposes. In addressing this problem, appropriated computational codes and nuclear data should be used. Since the $90 \mathrm{~s}$, different burn-up calculation systems have been developed, coupling a neutron transport code with an isotopic inventory code. In this sense, the
Monte Carlo $N$-particle transport code MCNP (Briesmeister, 2000) and the one-group depletion code ORIGEN (NEA, 2002) have been linked in automatic running programs such as MCODE (Xu et al., 2002), MONTEBURNS (Poston and Trellue, 2002) or MCOR (Tippayakul et al., 2006). Other systems, like OCTOPUS (Oppe and Kuijper, 2004), can combine MCNP as spectrum calculator with either the ORIGEN or FISPACT (Forrest and Sublet, 2001) code as burn-up step calculator. Our system, MCNP-ACAB (García-Herranz et al., 2005) combines MCNP and the inventory code ACAB (Sanz, 2000). These computational methods are reported to be satisfactory for the calculation of the isotopic inventory. 
However, in order to have confidence in the results, the need is now accepted to estimate the uncertainties in the calculated inventory, as far as these uncertainties are caused by (i) uncertainties in the basic data, and (ii) approximations in the calculational models. Most of the above-mentioned code systems lack this capability when dealing with burn-up problems; only the OCTOPUS code system, by means of the CASEMATE code (Kuijper et al., 2005), and MCNP-ACAB, by means of the ACAB capabilities, are able to calculate the uncertainties in the final nuclide densities caused by uncertainties in activation cross-sections. But both neglect the effect of the statistical errors in comparison with the effect of cross-section errors.

In the frame of this problem, the purpose of this paper is to present a general methodology to propagate the uncertainties throughout the burn-up period when using a coupled Monte Carlo spectrum-depletion approach. The different error sources are indicated and the most contributing ones are within the scope of this paper: cross-sections uncertainties and statistical errors. The proposed methodology to evaluate the effects of error propagation is implemented in our MCNP-ACAB system and applied to a high burn-up benchmark exercise.

\section{Statement of the problem}

Let $N(t)=\left[N_{1}(t), N_{2}(t), \ldots, N_{\mathrm{M}}(t)\right]^{\mathrm{T}}$ be the nuclide composition of a material, consisting of $M$ different nuclides, at time $\mathrm{t}$. The set of differential equations which describe the evolution of $N$ in a neutron field may be written as:

$\frac{\mathrm{d} N}{\mathrm{~d} t}=\boldsymbol{A} N=[\lambda] N+\left[\boldsymbol{\sigma}^{\mathrm{eff}}\right] \Phi N$

where $\boldsymbol{A}$ is the transition matrix, $[\lambda]$ is the $M$-by- $M$ matrix involving the decay values, $\left[\boldsymbol{\sigma}^{\mathrm{eff}}\right]$ is the matrix involving the one-group effective cross-sections, and $\Phi$ is the space-energy integrated neutron flux. Given $N_{0}=N(0)$ the initial nuclide density vector, the solution is

$N(\mathbf{t})=\exp (\boldsymbol{A} \mathbf{t}) N_{0}$

assuming a constant spectrum (hence constant effective one-group cross-sections) and a constant flux over the entire time step $[0, t]$.

In nuclear systems where the changes in the nuclide composition influences the neutron flux distribution, a sequence of combined flux-spectrum and depletion calculations are to be done. In such combined calculations, the whole burn-up period is divided into several consecutive time intervals. For each time interval, a transport calculation is carried out and the evaluated reaction rates are used in solving the burn-up Eq. (1) to obtain the inventory at the end of the time interval.

Our goal is not only to compute the vector $N$ of nuclide compositions along time, but also to estimate how the different sources of uncertainties resulting from the complex spectrum-burn-up scheme are propagated to $N$. Let us start analyzing the sources of uncertainties in this kind of combined calculations.

\subsection{Sources of uncertainties in a depletion calculation}

Assuming no uncertainties in the initial nuclide densities, uncertainties can be found in all the parameters involved in $\mathrm{Eq}$. (1), that is, in decay constants $\lambda$, one-group effective cross-sections $\sigma^{\text {eff }}$, and space-energy integrated neutron flux $\Phi: \quad N=N\left(\lambda, \sigma^{\text {eff }}, \Phi\right) \Rightarrow \Delta_{N}$ depends on $A_{\lambda}, \Delta_{\sigma^{\text {eff }}}, A_{\Phi}$, where $A$ denotes the uncertainty or relative error.

1. Uncertainties in decay constants $\Delta_{\lambda}$ can be taken, when existing, from the evaluated nuclear data libraries.

2. Uncertainties in one-group effective cross-sections $\Delta_{\sigma^{\mathrm{eff}}}$ depend on both uncertainties in the evaluated nuclear cross-section data $\Delta_{\sigma^{g}}$ and uncertainties in the flux spectrum $\Delta_{\phi^{g}}(E)$ obtained from a stochastic transport calculation, since $\sigma^{\text {eff }}=\sum_{g} \sigma^{g} \phi^{g} / \sum_{g} \phi^{g}$.

The uncertainties in the evaluated cross-sections can be found in two types of libraries: activation-oriented nuclear data libraries and general purpose evaluated nuclear data files. A review of the nuclear data uncertainties available in the most recent internationally distributed nuclear data libraries was recently performed (Sanz et al., 2006), and it showed: (i) there is a lack of variance covariance data of relevant nuclides; (ii) validity of variance-covariance data is under discussion. Then, results using those data should be regarded as a kind of "proof of principle"; calculations should be repeated once better data becomes available. On the other hand, uncertainties in the flux spectrum are a result of the uncertainties in transport cross-sections, densities (in general, in all the input data needed for the transport calculation) and of the statistical nature of the Monte Carlo neutron transport calculation (if, like in our case, a stochastic transport code is used to perform the spectrum calculations).

3. Uncertainties in the integrated neutron flux, $\Delta_{\Phi}$. In order to obtain the flux level, a normalization factor is required. Generally, such factor is assumed to be the constant power, that is, there is a control mechanism that will change/compensate the flux level in order to maintain the requested constant power level. If $P$ denotes the total fission power, $P=K N \sigma_{\mathrm{f}} \Phi V$, being $V$ the volume of material zone, $N \sigma_{\mathrm{f}} \Phi$ the fission rate and $K$ the conversion factor. From this equation, it can be seen that the uncertainty in the integrated neutron flux will depend on the uncertainties in the isotopic concentration and uncertainties in the one-group fission cross-sections of the fissile material.

In summary, the sources of uncertainty in a depletion calculation can be classified into: (i) uncertainties in basic input nuclear data; (ii) uncertainties due to the statistical nature of Monte Carlo neutron transport calculation; (iii) uncertainties introduced by the normalization factor: $N=N\left(\lambda, \sigma^{\text {eff }}, \Phi\right)=N\left(\lambda, \sigma^{g}, \phi^{g}(E), \Phi\right)$. 


\subsection{Assumptions and objectives}

Most of reported codes to propagate uncertainties to the isotopic inventory and associated parameters only account for the influence of uncertainties in basic crosssection data (Sanz, 2000; Kuijper et al., 2005). One way to evaluate the influence of flux normalization was briefly stated by Ivanov (2005). The influence of statistical uncertainties has been recently investigated in deep by Tohjoh et al. (2006). Their results reveal that the propagated statistical errors on the nuclide densities in Monte Carlo burn-up calculations are low up to $60 \mathrm{GWd} / \mathrm{t}$. However, they do not evaluate the propagated errors for higher burn-up (exceeding $100 \mathrm{GWd} / \mathrm{t}$ ) and they do not consider the combined effect with cross-section errors. The combined effect of both cross-section and flux errors was studied by Takeda et al. (1999) by using a sensitivity method. For fast reactors, they concluded that it is not necessary to consider the statistical errors (since they are smaller than errors of the cross-section libraries), but that their effect might be large in thermal reactors and should be analyzed. Furthermore, the propagation along burn-up was not studied.

In this work, we investigate the influence of uncertainties in the activation cross-sections and statistical errors in the neutron flux spectrum on the calculated actinide inventory along burn-up for any kind of nuclear systems. In other words, we are concerned with the propagation to the nuclide densities, as calculated at final time, of the uncertainties in one-group effective cross-sections. For simplicity, we assume that we have a single homogeneous material, of which the evolution of the composition is to be calculated.

The following assumptions are made:

(i) The influence of uncertainties in decay constants, fission yields and other input parameters different from the cross-sections is of minor importance, which tends to be true for actinides.

(ii) No uncertainties in the integrated neutron flux are considered, that is, the integrated neutron flux is taken as the normalization factor.

(iii) The flux spectrum is not sensitive to uncertainties in cross-sections and densities. That is, we will assume that the uncertainties in the transport input data lead to considerably smaller errors in the flux spectrum than the statistical fluctuations, so that our formalism will not take into account the cross-section error propagation within the transport calculation.

\section{Methodologies to propagate uncertainties on a coupled Monte Carlo spectrum-depletion approach}

In this section we address the topic of the methodologies to be applied to estimate uncertainty propagation to the isotopic inventory in Monte Carlo depletion calculations.
It is useful to bear in mind the coupled calculation scheme to infer an error propagation procedure throughout the time.

After dividing the whole burn-up period into several consecutive time intervals, the coupled scheme consists of:

(a) Calculating the neutron flux distribution in a fixed step (transport code). In this work we assume that a Monte Carlo code is used.

(b) Collapsing the effective total one-group cross-sections and calculating the integrated flux making use of a normalizing coefficient (linkage program).

(c) Calculating the nuclide evolution through Eq. (2) assuming constant flux and constant one-group microscopic cross-sections until the next time step (depletion code) and return to (a).

The same sequence should be followed to propagate the errors. Step (a) would propagate all the uncertainties in the transport input data on the neutron flux. Since a Monte Carlo transport code is used, there will also be inevitable statistical errors. Then, the errors in the reaction rates (consequence of the uncertainties in cross-sections and errors in the neutron field) as well as uncertainties in decay constants should be propagated on the nuclide inventory in step (c). Then, in the next time interval, the errors in the calculated nuclide concentrations and in the rest of transport input data should be propagated in the subsequent neutron calculation, and so on. In this way, all uncertainties existing at the beginning of time would be propagated to the end of cycle.

\subsection{Uncertainty propagation by a "brute force" random sampling method}

A first methodology to perform uncertainty analysis would be random simulation or "brute force" Monte Carlo method. The multi-step scheme of stochastic neutronics and burn-up could be regarded as a single process with input parameters (nuclear data) and output (final densities). This scheme should be run many times; for each run, a simultaneous random sampling of the probability density functions (PDF) of all the input parameters should be carried out, and the output parameters would be obtained (see Fig. 1). Obviously, the transport code should be able to sample the PDF of all the input nuclear data involved in the whole problem. A statistical analysis of the results would allow to assess the uncertainties in the calculated densities.

The advantages of this "brute force methodology" is that inherently would propagate the uncertainties in cross-sections and densities within the Monte Carlo transport calculation (since for each time, the spectrum calculation is carried out taken the actual calculated densities as input), as well as the uncertainties due to the statistical nature of the spectrum calculation. However, 


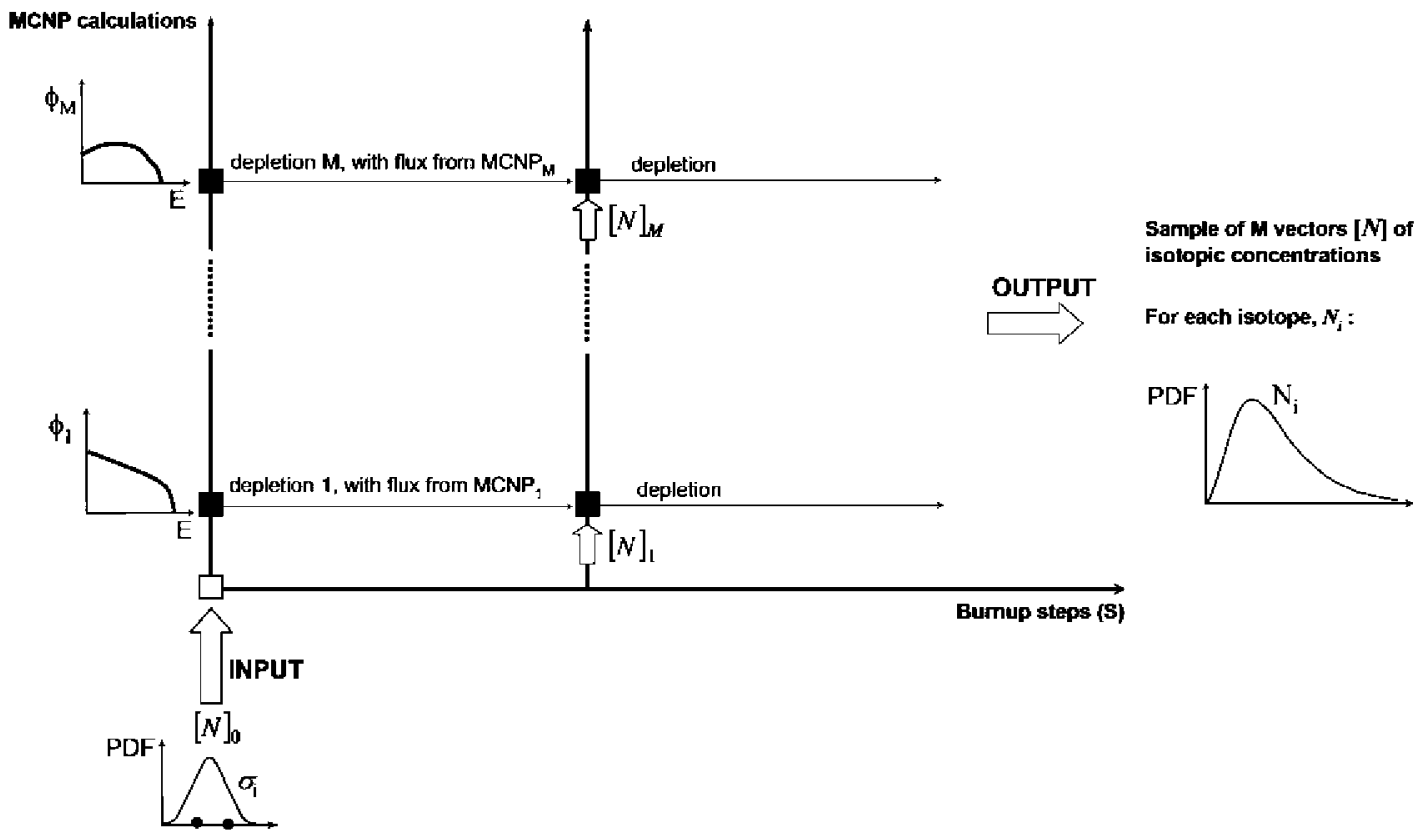

Fig. 1. "Brute-force" Monte Carlo method scheme to propagate uncertainties in final densities.

the methodology is impractical, because it would take a very long time to run, due to the large number of Monte Carlo transport calculations needed. To obtain a sample of $M$ vectors of isotopic concentrations in a problem with $S$ burn-up steps, $M \cdot S$ MCNP runs would be needed.

\subsection{Uncertainty propagation by a sensitivity method}

Another kind of methodology to propagate errors in nuclide densities is the linear sensitivity analysis. This method is implemented in several codes, such as ACAB or CASEMATE, but in both cases only the uncertainties in activation cross-sections are propagated along the consecutive spectrum-depletion steps. That is, it is assumed that the statistical nature of flux spectrum calculation is of minor importance.

The question now is how the statistical fluctuations together with the cross-section uncertainties affect the computed isotopic inventory. Let us consider now the influence of both sources of error.

For a fixed burn-up step $s$ (time interval $\left[t_{s}, t_{s+1}\right]$ ), the multigroup flux spectrum (represented by a random vector $\phi=\left[\phi^{1}, \ldots \phi^{g}, \ldots \phi^{G}\right]^{\mathrm{T}}$ ) is calculated and the $R$ multigroup microscopic cross-sections (each one represented by $\sigma_{j}=\left[\begin{array}{lll}\sigma_{j}^{1}, & \ldots \sigma_{j}^{g}, \quad \ldots \sigma_{j}^{G}\end{array}\right]^{\mathrm{T}}$ ) are collapsed to yield the set of one-group effective cross-sections $\sigma^{\text {eff }}=\left[\begin{array}{lll}\sigma_{1}^{\text {eff }} & \ldots \sigma_{j}^{\text {eff }} \text {, }\end{array}\right.$ $\left.\ldots \sigma_{R}^{\text {eff }}\right]^{\mathrm{T}}$. Let us assume the flux spectrum normalized to unity so that $\sigma_{j}^{\text {eff }}=\sum_{g} \sigma_{j}^{g} \phi^{g}=\phi^{\mathrm{T}} \sigma_{j}$. The error in this one-group cross-section is composed of two terms: uncertainties in microscopic multi-group cross-sections and statistical errors in the flux spectrum.
Each concentration $N_{i}$ at the end of the burn-up step $s$ obtained from Eq. (1) is a function of the one-group effective cross-sections, $N_{i}=N_{i}\left(\sigma^{\text {eff }}\right)$, because the other parameters of the equation are constant by hypothesis. Let us assume that $\hat{\sigma}^{\text {eff }}$ is the best-estimated one-group cross-section vector and $N_{i}\left(\hat{\sigma}^{\text {eff }}\right)$ the solution of Eq. (1) at this point. Taylor series provides a means of approximating $N_{i}$ about $\hat{\sigma}^{\text {eff }}$ :

$N_{i}\left(\sigma^{\mathrm{eff}}\right)=N_{i}\left(\hat{\sigma}^{\mathrm{eff}}\right)+\sum_{j=1}^{R}\left[\frac{\partial N_{i}}{\partial \sigma_{j}}\right]_{\hat{\sigma}_{\text {eff }}}\left(\sigma_{j}^{\mathrm{eff}}-\hat{\sigma}_{j}^{\mathrm{eff}}\right)+\cdots$

where $\left[\frac{\partial N_{i}}{\partial \sigma_{j}}\right]$ is known as the sensitivity coefficient. The random variable $\varepsilon_{j}=\sigma_{j}^{\text {eff }}-\hat{\sigma}_{j}^{\text {eff }}$ is the error in the one-group effective cross-section for reaction $j$ in the burn-up step. Since $\sigma_{j}^{\text {eff }}=\sum_{g} \sigma_{j}^{g} \phi^{g}$, the error can be expressed as:

$\varepsilon_{j}=\sum_{g=1}^{G} \phi^{g}\left(\sigma_{j}^{g}-\hat{\sigma}_{j}^{g}\right)+\sum_{g=1}^{G} \sigma_{j}^{g}\left(\phi^{g}-\hat{\phi}^{g}\right)=\phi^{\mathrm{T}} \varepsilon_{\sigma_{j}}+\sigma_{j}^{\mathrm{T}} \varepsilon_{\phi}$

where $\varepsilon_{\sigma_{j}}$ and $\varepsilon_{\phi}$ are the random vectors of errors due to uncertainties in the multigroup cross-sections and due to errors in the multigroup flux spectrum respectively. A measure of the uncertainty in those vectors is their variance. For the random vector $\varepsilon_{\sigma_{j}}$, the $G$-by- $G$ covariance matrix $\left[\mathrm{COV}_{\sigma_{j}}\right]$ can be processed directly from the uncertainty information included in nuclear data libraries, and for the random vector $\varepsilon_{\phi}$, the $G$-by- $G$ covariance matrix $\left[\mathrm{COV}_{\phi}\right]$ can be obtained from a single MCNP calculation, as it will be demonstrated further. 


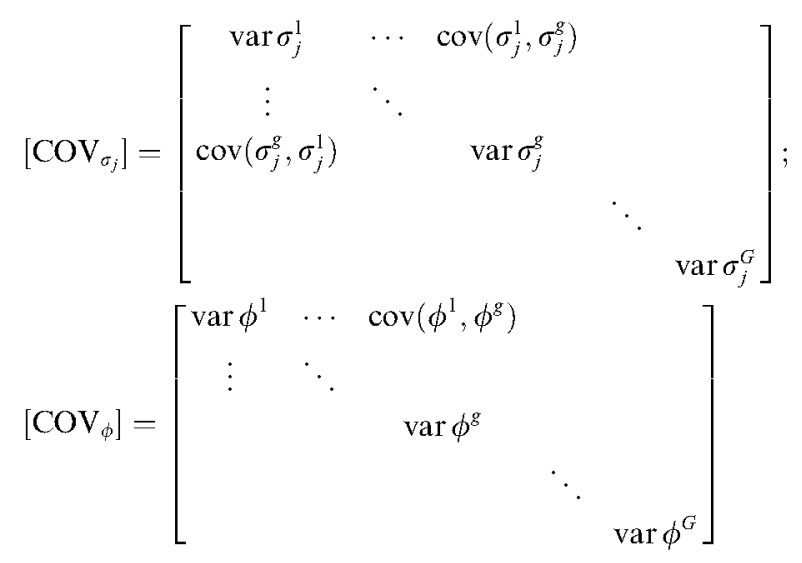

Eq. (3) gives a direct method for obtaining the variation in the concentrations of the $M$ nuclides: $N\left(\sigma^{\text {eff }}\right)-N\left(\hat{\sigma}^{\text {eff }}\right) \approx \boldsymbol{S} \varepsilon$, where $\boldsymbol{S}$ denotes the $M$-by- $R$ matrix containing the sensitivity coefficients of the isotopic concentrations with respect to the one-group cross-sections.

$\boldsymbol{S}=\left[\begin{array}{cccc}\frac{\partial N_{1}}{\partial \sigma_{1}} & \cdots & \frac{\partial N_{1}}{\partial \sigma_{j}} & \cdots \\ \vdots & \ddots & & \\ \frac{\partial N_{i}}{\partial \sigma_{1}} & & \ddots & \vdots \\ \vdots & \cdots & & \frac{\partial N_{M}}{\partial \sigma_{R}}\end{array}\right]$

The variance of the nuclide concentrations can be evaluated as follows ( $E$ means expectation):

$\operatorname{var} N=E\left[(N-\widehat{N})^{2}\right] \approx \boldsymbol{S}\left[\mathrm{COV}_{\sigma^{\text {eff }}}\right] \boldsymbol{S}^{\mathrm{T}}$

where $\left\lfloor\mathrm{COV}_{\sigma^{\mathrm{eff}}}\right\rfloor$ is the $R$-by- $R$ covariance matrix of the one-group effective cross-sections, hereafter referred as effective covariance matrix:

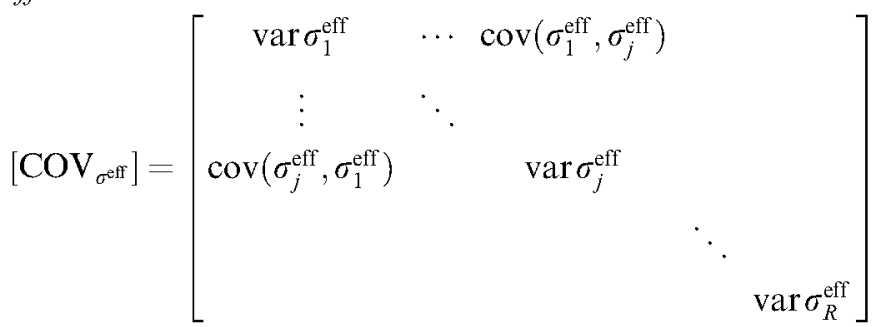

The $j-k$ element of that matrix can be calculated as follows:

$$
\begin{aligned}
\operatorname{cov}\left(\sigma_{j}^{\mathrm{eff}}, \sigma_{k}^{\mathrm{eff}}\right)= & E\left[\varepsilon_{j} \varepsilon_{k}^{\mathrm{T}}\right]=\hat{\phi}^{\mathrm{T}} E\left[\varepsilon_{\sigma_{j}} \varepsilon_{\sigma_{k}}^{\mathrm{T}}\right] \hat{\phi}+\hat{\phi}^{\mathrm{T}} E\left[\varepsilon_{\sigma_{j}} \varepsilon_{\phi}^{\mathrm{T}}\right] \hat{\sigma}_{k} \\
& +\hat{\sigma}_{j}^{\mathrm{T}} E\left[\varepsilon_{\phi} \varepsilon_{\sigma_{k}}^{\mathrm{T}}\right] \hat{\phi}+\hat{\sigma}_{j}^{\mathrm{T}} E\left[\varepsilon_{\phi} \varepsilon_{\phi}^{\mathrm{T}}\right] \hat{\sigma}_{k}
\end{aligned}
$$

It is reasonable to assume that there is no correlation between the cross-section uncertainties and the Monte Carlo statistical errors $\left(E\left[\varepsilon_{\sigma_{j}} \varepsilon_{\phi}^{\mathrm{T}}\right]=0\right)$. Then, the diagonal terms of the matrix can be written as:

$$
\begin{aligned}
\operatorname{var} \sigma_{j}^{\mathrm{eff}} & =E\left[\varepsilon_{j}^{2}\right]=\hat{\phi}^{\mathrm{T}}\left[\operatorname{COV}_{\sigma_{j}}\right] \hat{\phi}+\hat{\sigma}_{j}^{\mathrm{T}}\left[\operatorname{COV}_{\phi}\right] \hat{\sigma}_{j} \\
& =\left.\operatorname{var} \sigma_{j}^{\mathrm{eff}}\right|_{\sigma}+\left.\operatorname{var} \sigma_{j}^{\mathrm{eff}}\right|_{\phi}
\end{aligned}
$$

Eq. (5) shows a method to compute the effect of the statistical errors together with the multigroup cross-section uncertainties on the nuclide concentrations, by calculating the standard sensitivity coefficient matrix (the same coefficients computed by the depletion codes that take into account the uncertainties in activation cross-sections but without considering statistical errors) and an effective covariance matrix.

Let us assume moreover that the cross-section errors of different reactions are uncorrelated $\left(E\left[\varepsilon_{\sigma_{i}} \varepsilon_{\sigma_{k}}^{\mathrm{T}}\right]=0\right)$, as supposed in the activation data libraries. Then, the off-diagonal terms are:

$\operatorname{cov}\left(\sigma_{j}^{\mathrm{eff}}, \sigma_{k}^{\mathrm{eff}}\right)=\hat{\sigma}_{j}^{\mathrm{T}} E\left[\varepsilon_{\phi} \varepsilon_{\phi}^{\mathrm{T}}\right] \hat{\sigma}_{k}=\hat{\sigma}_{j}^{\mathrm{T}}\left[\mathrm{COV}_{\phi}\right] \hat{\sigma}_{k}$

That means that, even if the correlations among multigroup cross-sections for different reactions are neglected, the one-group effective cross-sections are correlated when considering the errors in the flux spectrum.

However, it can be demonstrated that such correlation factor is limited:

$$
\begin{gathered}
\operatorname{corr}\left(\sigma_{j}^{\mathrm{eff}}, \sigma_{k}^{\mathrm{eff}}\right)=\frac{\operatorname{cov}\left(\sigma_{j}^{\mathrm{eff}}, \sigma_{k}^{\mathrm{eff}}\right)}{\sqrt{\operatorname{var} \sigma_{j}^{\mathrm{eff}}} \sqrt{\operatorname{var} \sigma_{k}^{\mathrm{eff}}}} \\
=\frac{\hat{\sigma}_{j}^{\mathrm{T}}\left[\mathrm{COV}_{\phi}\right] \hat{\sigma}_{k}}{\sqrt{\left.\operatorname{var} \sigma_{j}^{\mathrm{eff}}\right|_{\sigma}+\left.\operatorname{var} \sigma_{j}^{\mathrm{eff}}\right|_{\phi}} \sqrt{\left.\operatorname{var} \sigma_{k}^{\mathrm{eff}}\right|_{\sigma}+\left.\operatorname{var} \sigma_{k}^{\mathrm{eff}}\right|_{\phi}}} \\
\text { Since } \hat{\sigma}_{j}^{\mathrm{T}}\left[\operatorname{COV}_{\phi}\right] \hat{\sigma}_{k} \leqslant \sqrt{\hat{\sigma}_{j}^{\mathrm{T}}\left[\operatorname{COV}_{\phi} \hat{\sigma}_{j}\right.} \sqrt{\hat{\sigma}_{k}^{\mathrm{T}}\left[\operatorname{COV}_{\phi}\right] \hat{\sigma}_{k}} \text { then }, \\
\operatorname{corr}\left(\sigma_{j}^{\mathrm{eff}}, \sigma_{k}^{\mathrm{eff}}\right) \leqslant \frac{1}{\sqrt{k_{j}^{2}+1} \sqrt{k_{k}^{2}+1}} \text { where } k_{j}=\frac{\left.\operatorname{var} \sigma_{j}^{\mathrm{eff}}\right|_{\sigma}}{\left.\operatorname{var} \sigma_{j}^{\mathrm{eff}}\right|_{\phi}}
\end{gathered}
$$

For example, if $k_{j} \approx k_{k} \approx 10$, then $\operatorname{corr}\left(\sigma_{j}^{\text {eff }}, \sigma_{k}^{\text {eff }}\right) \leqslant 1 / 101$. Then, if the uncertainties in the one-group effective crosssections due to the multigroup cross-section errors are much larger than the uncertainties due to the flux errors, the correlation factors are negligible, and the off-diagonal elements of the effective covariance matrix can be set to zero. In such case, Eq. (5) can be written as follows:

$$
\begin{aligned}
& \operatorname{var} N \approx \boldsymbol{S}\left[\mathrm{COV}_{\sigma^{* f f}}\right] \boldsymbol{S}^{\mathbf{T}} \\
& \approx S\left\{\left[\begin{array}{ccc}
\ddots & 0 & \\
0 & \hat{\phi}^{\mathrm{T}}\left[\mathrm{COV}_{\sigma_{j}} \mid \hat{\phi}\right. & \\
& & \ddots
\end{array}\right]+\left[\begin{array}{lll}
\ddots & 0 & \\
0 & \hat{\sigma}_{j}^{\mathrm{T}}\left[\mathrm{COV}_{\phi}\right] \hat{\sigma}_{j} & \\
& &
\end{array}\right]\right\} \boldsymbol{S}^{\mathrm{T}}
\end{aligned}
$$

That is, the effective covariance matrix can be computed summing up two matrices:

- The first one propagates the multigroup cross-section uncertainties when there is no statistical flux errors (and correlations among different reactions are neglected).

- The second one propagates only statistical flux errors when there is no multigroup cross-section uncertainties/covariances.

The generalized sensitivity formulation represented by Eq. (8) has been implemented in $\triangle \mathrm{CAB}$ and applied to a 
HTR benchmark problem in Section 5. To propagate uncertainties in cross-sections, only the first term of the effective covariance matrix is computed; to propagate flux statistical errors, only the second term is computed, and to propagate both kind of errors, both terms of the effective covariance matrix are summed up.

The procedure followed in the combined neutronics and burn-up schemes to propagate uncertainties by this sensitivity formalism is shown in Fig. 2. The most important limitations of this sensitivity method are: first, that it is impractical to deal with the global effect of the uncertainties of the complete set of cross-sections; and second, the analysis based on a first order Taylor approximation does not allow to account for non-linear effects and is expected to fail when the uncertainties are high.

\subsection{Uncertainty propagation by a hybrid Monte Carlo method}

To overcome some of the limitations of the two previous methodologies, we propose a Monte Carlo uncertainty method that is a hybrid form between them. This methodology, implemented in the $\mathrm{ACAB}$ code and shown in Fig. 3, accounts for the impact of activation cross-section uncertainties and flux spectrum errors along the consecutive spectrum-depletion steps as follows:

- In a first step, a coupled neutron-depletion calculation is carried out only once, taken the best-estimated values for all the parameters involved in the problem. That is, when solving the transport equation to calculate the flux distribution for each time step, nor uncertainties in the input parameters nor statistical fluctuations are taken into account. This is called the best-estimated multi-step calculation.

- In a second step, the uncertainty analysis to evaluate the influence of the uncertainties in the flux and in the crosssections involved in the transmutation process on the isotopic inventory is accomplished by the ACAB code. It performs a simultaneous random sampling of the probability density functions (PDF) of all those variables. Then, ACAB computes the isotopic concentrations at the end of each burn step, taking the fluxes halfway through each burn step determined in the best-estimated calculation. In this way, only the depletion calculations are repeated or run many times. A statistical analysis of the results allow to assess the uncertainties in the calculated densities. To obtain a sample of $M$ vectors of isotopic concentrations in a problem with $S$ burn-up steps, only $S$ runs with MCNP are needed.

To apply random simulation, the PDF of the involved variables have to be known.

\subsubsection{Propagating uncertainties in cross-sections}

If we are interested in propagating only uncertainties in cross-sections, different assumptions can be made for the PDF. The simplest and more usual (in many other areas) is the normal distribution, but when the variance is large, this distribution can generate negative values for the cross-sections. To avoid this drawback an alternative distribution is the log normal, that is:

$\log \left(\frac{\sigma}{\hat{\sigma}}\right) \rightarrow N(0, A)$ (normal of mean zero and standard deviation $\Delta$ )

where $\hat{\sigma}$ is a best-estimate cross-section read from a given library and $A$ is its relative error included in the corresponding uncertainty library. There are quite a few important reasons to recommend this well known distribution. For instance, taking into account that $\log \left(\frac{\sigma}{\sigma}\right)=\log$ $\left(1+\frac{\sigma-\hat{\sigma}}{\hat{\sigma}}\right) \approx \frac{\sigma-\hat{\sigma}}{\hat{\sigma}}$, when $\frac{\sigma-\hat{\sigma}}{\hat{\sigma}}$ is small (that is, when $\Delta$ is small), the log normal assumption is practically equivalent to the normality.

In multigroup uncertainty libraries, the relative error $A$ of the cross-section in each particular energy group $g$ is provided. Then, the above distribution is applied to each multigroup cross-section $\sigma_{j}^{g}$. If a full cross-section covariance matrix were given in the uncertainty library, the next joint probability distribution would be assumed:

$\log \left(\begin{array}{c}\sigma_{j}^{1} / \hat{\sigma}_{j}^{1} \\ \vdots \\ \sigma_{j}^{G} / \hat{\sigma}_{j}^{G}\end{array}\right) \rightarrow N\left(0, \mathrm{COVR}_{\sigma_{j}}\right)$

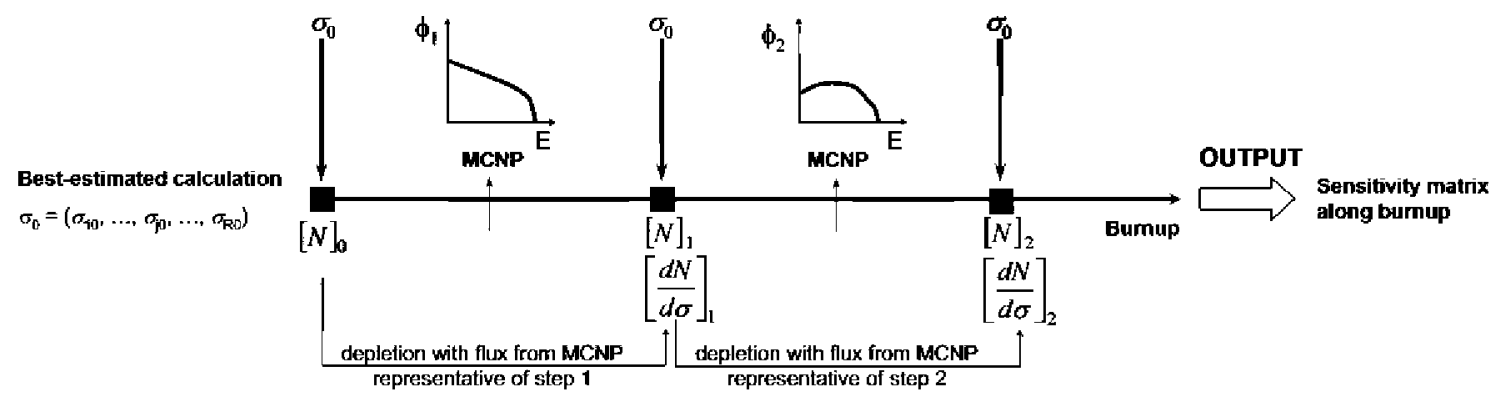

Fig. 2. Sensitivity/uncertainty method to propagate uncertainties in final densities. 

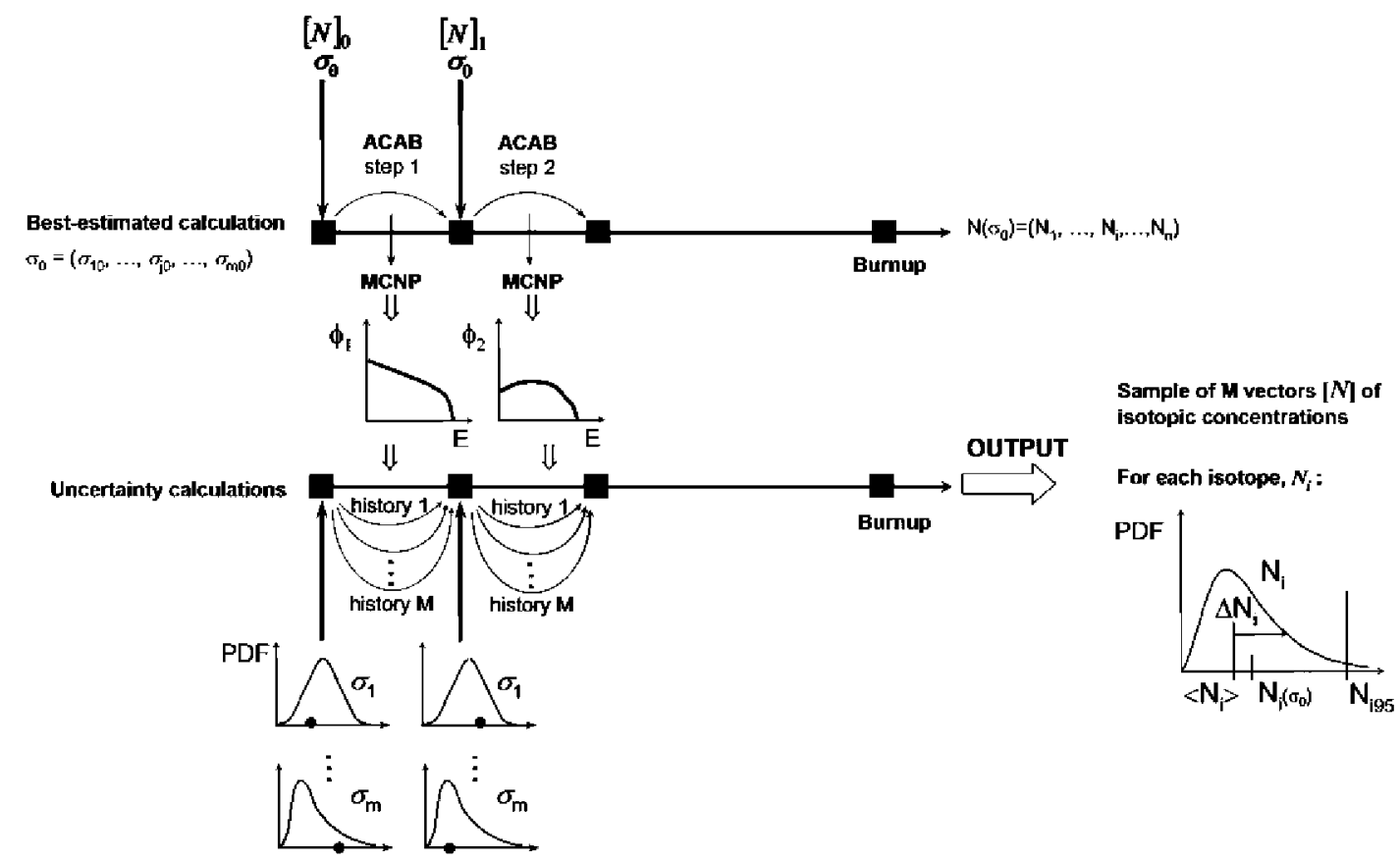

Fig. 3. Hybrid Monte Carlo method scheme implemented in MCNP-ACAB to propagate uncertainties in final densities.

Sampling the adequate probability distribution, a sample of the spectrum-averaged cross-section $\sigma_{j}^{\text {eff }}=\hat{\phi}^{\mathrm{T}} \sigma_{j}$ is obtained. From the random vector of one-group effective cross sections $\sigma^{\text {eff }}=\left[\begin{array}{lll}\sigma_{1}^{\text {eff }}, \ldots \sigma_{j}^{\text {eff }} & \ldots \sigma_{R}^{\text {eff }}\end{array}\right]^{\mathrm{T}}$, the matrix $\boldsymbol{A}$ (of Eq. (1)) is computed and the vector of nuclide quantities is obtained. Repeating this sequence, it is possible to get a sample of $M$ vectors of nuclide quantities and, from the sample, to estimate the mean, variance, etc. of the nuclide distribution.

\subsubsection{Propagating fux statistical errors}

To consider the influence of the statistical fluctuations, a PDF for the flux spectrum has to be assumed. And this is not obvious because of two reasons:

(i) From a MCNP calculation, for each tally (fluxes e.g.) the mean value and the relative error are known. The calculated variances for the tallies assume that all the neutron histories are independent. But, in a criticality calculation, the location of fission sites in one generation are correlated with the locations of fission sites in successive generations. Then, the cycle-to-cycle estimates of the flux can be correlated, and because of such a correlation between histories, the variances given in the output file can be underestimated.

(ii) No correlations between energy groups are known, and a priori there are no reasons to think that different energy group fluxes are not strongly correlated.

Then, in order to analyze the purely statistical variation of the neutron flux, and infer an appropriate PDF, several independent MCNP calculations (using different random number seed) should be run. By subsequently performing a statistical analysis on the calculated flux spectra, the covariance matrix can be estimated.

This analysis has been carried out in Section 5.3 for the high burn-up benchmark problem used in this work. It will be demonstrated that the flux spectrum fits a normal distribution $\phi^{g} \rightarrow N\left(\hat{\phi}^{g}, \hat{s}\left(\hat{\phi}_{g}\right)\right)$, where the standard deviation of the normal is the flux statistical error directly taken from a single MCNP calculation. On the other hand, no correlation between energy groups are seen. Then, a covariance matrix with no correlation between groups and the statisti$\mathrm{cal}$ variances in the diagonal can be made, $\left[\mathrm{COV}_{\phi}\right]$.

Instead of sampling such a covariance matrix to obtain samples of the neutron flux spectrum and then compute the spectrum-averaged cross-sections, it is possible to compute the variance of those effective cross-sections:

$\left.\operatorname{var} \sigma_{j}^{\mathrm{eff}}\right|_{\phi}=\hat{\sigma}_{j}^{\mathrm{T}}\left[\mathrm{COV}_{\phi}\right] \hat{\sigma}_{j}$

and to sample them using the following PDF:

$\sigma_{j}^{\mathrm{eff}} \rightarrow N\left(\hat{\sigma}_{j}^{\mathrm{eff}}, \sqrt{\left.\operatorname{var} \sigma_{j}^{\mathrm{eff}}\right|_{\phi}}\right)$

where $\hat{\sigma}_{j}^{\text {eff }}$ is the best-estimate value. This can be applied to get a sample of the random vector $\sigma_{\text {eff }}$ of cross-sections. From this, the sample of $M$ vectors of nuclide quantities can be computed. In the case of large flux spectrum errors, the normal distribution could generate negative values for the one-group effective cross-sections; then, a log-normal distribution could be assumed instead.

The above can be applied to obtain the one-group effective cross-sections or cross-sections in a given energy-group structure. 
3.3.3. Propagating both cross-section uncertainties and flux statistical errors

On one hand, for each cross-section $\sigma_{j}^{g}$, we assume a lognormal PDF, being $g$ the number of energy groups in which the cross-section relative errors $A_{\sigma}^{g}$ are given in the uncertainty library. Then, the variance of each cross-section due to the cross-section uncertainties is known: $\left.\operatorname{var} \sigma_{j}^{g}\right|_{\sigma}=\left(\Lambda_{\sigma}^{g}\right)^{2} \cdot\left(\hat{\sigma}_{j}^{g}\right)^{2}$.

On the other hand, the relative errors in the flux spectrum given by MCNP are used to compute, in the same energy-group structure, the variance of the cross-section in each group due to the flux deviations: $\left.\operatorname{var} \sigma_{j^{g}}\right|_{\phi}=$ $\sum_{g^{\prime} \in g}\left(\hat{\sigma}_{j}^{g^{\prime}}\right)^{2} \operatorname{var} \phi^{g^{\prime}}$.

Taking into account that $\operatorname{var} \sigma_{j}^{g}=\left.\operatorname{var} \sigma_{j}^{g}\right|_{\sigma}+\left.\operatorname{var} \sigma_{j}^{g}\right|_{\phi}$, we compute the variance of the cross-sections in the energygroup structure defined in the cross-section uncertainty library and we perform a simultaneous random sampling of all the variables using the PDF $\log \left(\sigma_{j}^{g} / \hat{\sigma}_{j}^{g}\right) \rightarrow N(0$, $\left.\sqrt{\operatorname{var} \sigma_{j}^{g}} / \hat{\sigma}_{j}^{g}\right)$, to get a sample of the random vector of cross-sections. From these vectors, a sample of nuclide concentrations is computed.

A full application of this procedure is in Section 5, and results will be compared with the ones obtained by using the sensitivity technique.

\section{HTR plutonium cell burn-up benchmark: MCNP-ACAB performance with no uncertainties}

\section{1. $M C N P-A C A B$ code}

The methodology of the MCNP-ACAB coupling procedure is described as follows (Fig. 4). MCNP calculates the neutron flux spectrum $(\phi(E))$ and effective total one-group cross-sections ( $\sigma_{\mathrm{MCNP}}^{\mathrm{eff}}$ ) for the number of isotopes and reactions specified in the Monte Carlo input. The activation cross-sections for the rest of reactions and the rest of nuclides not included in the MCNP but considered in ACAB are obtained by collapsing the extended activation crosssection library (temperature-dependent, such as ENDF/ B-6, JENDL-3.3, or processed for a given temperature, such as EAF2005 [300 K]) with the MCNP flux. A similar procedure is used to obtain the effective fission yields starting from the JEF-2.2 fission yield library. For nuclides with cross-sections leading to meta stable states, $(n, \gamma-m)$ and $(n, 2 n-m)$, a branching ratio is used to update the ACAB cross-section library from total one-group MCNP values. This ratio is the same as in the extended activation crosssection library.

With the resulting spectrum-dependent libraries (activation cross-section $\sigma_{\mathrm{ACAB}}^{\text {eff }}$ and fission yields $\langle\gamma\rangle$ ) and with the extended decay library, ACAB computes the isotopic inventory and feedbacks the resulting material compositions to MCNP. It is not practical to perform a MCNP calculation for all nuclides considered in the depletion code due to the excessive CPU time demanded and the unavailability of many MCNP cross-sections. Therefore, only the isotopes with influence on the reactivity and neutron spectrum are feed back into the Monte Carlo input. The coupling MCNP-ACAB is carried out using a middle-time step approach.

Another important feature of ACAB, although not used in this work, is the capability to compute a number of quantities useful to perform safety and waste management assessments. This is done by using appropriate available or on purpose generated radiological/dosimetric libraries. As examples, ACAB can compute the decay heat which is useful in safety assessments, and regarding waste management, it can compute waste disposal ratings/indexes for the shallow land burial and clearance options. Much more additional quantities can be obtained (Sanz, 2000).

As far as fusion applications, the potential of ACAB to predict the isotopic inventory and to estimate uncertainties has been proved in an extensive number of benchmark and studies (Sanz et al., 2003, 2004; Cabellos et al., 2006). ACAB has also been satisfactorily applied in core burnup calculations on Accelerator Driven Systems (Cabellos et al., 2005) and recently in a PWR pin-cell benchmark (García-Herranz et al., 2005). In this work, the High Temperature gas-cooled Reactor (HTR) Plutonium Cell Burnup Benchmark defined in Kuijper et al. (2004) has been chosen.

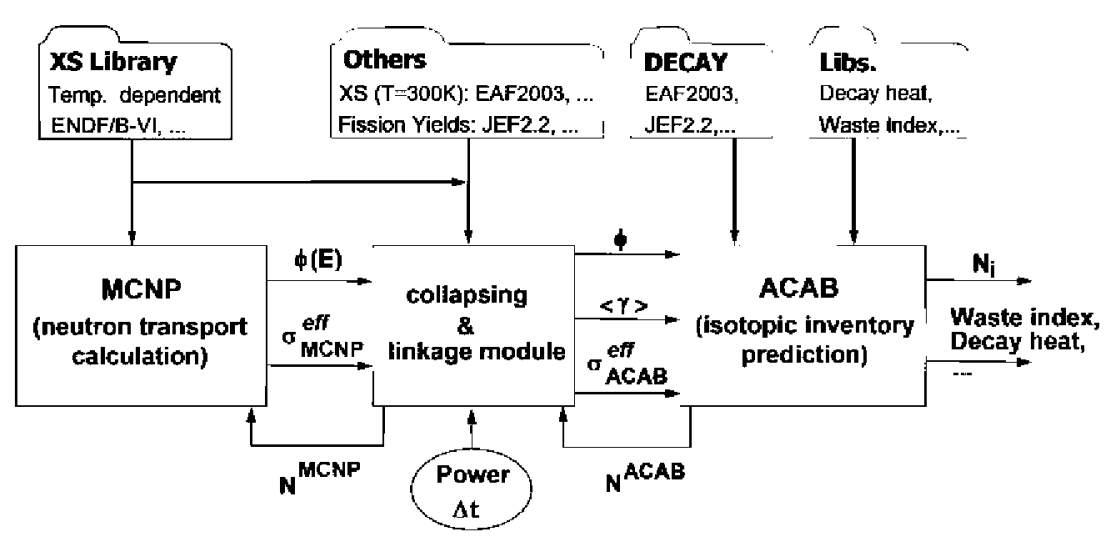

Fig. 4. MCNP-ACAB coupling procedure for each time step. 


\subsection{Benchmark description}

This calculational exercise was defined to obtain a validation of several code systems to be used for the analysis of HTR for plutonium burning applications. The benchmark concerns a spherical HTR ("pebble") fuel element containing coated $\left(\mathrm{PuO}_{2}\right)$ fuel particles. The neutronic boundary condition is assumed to be white. In total, four different benchmark cases were defined, characterized by different initial isotopic compositions of Pu. The case "Cl", with $1.5 \mathrm{~g} \mathrm{Pu}$ per fuel element obtained from reprocessed LWR MOX fuel (called second generation $\mathrm{Pu}$ ), is the one considered in this work. The fuel element parameters and isotopic compositions are specified in Kuijper et al. (2004).

The main requested calculations concerned the multiplication factor and isotopic composition during the irradiation of the fuel element at constant power of $1.0 \mathrm{~kW}$ (per fuel assembly) up to the unusually high burn-up of 800 $\mathrm{MWd} / \mathrm{kgHM}$. An irradiation time of 1200 full power days is required to reach the fixed burn-up.

Requested calculations were performed, among others, by NRG, employing the WIMS8A code and the OCTOPUS code system. Their main features are presented in Table 1. Results obtained with $\mathrm{MCNP}-\mathrm{ACAB}$ are benchmarked against NRG calculations.

\subsection{Results and analysis}

In the MCNP-ACAB calculations, the MCNP code used the JEFF-3.1 cross-section library at $1000 \mathrm{~K}$ to calculate the flux spectrum in 175 energy groups and the effective one-group cross-sections for the isotopes and reactions specified in the Monte Carlo input. Using the 175-group structure, the rest of cross-sections not available in the MCNP calculations were collapsed from the EAF2005 activation library. A similar collapsing procedure was used to obtain the effective fission yields starting from the JEF2.2 fission yield library. All these updated parameters were subsequently used by the ACAB inventory code.

The MCNP-ACAB system has been run under linux, in a 4 CPU cluster. The MCNP code (version 4C3) has been compiled in parallel with PVM. The sequence of alternating neutron flux spectrum and burn-up step calculations is being also parallelized. The complete calculation (50

Table 1

Summary of the main features of the code systems used by NRG

\begin{tabular}{lll}
\hline & NRG-WIMS & NRG-OCTOPUS \\
\hline $\begin{array}{l}\text { Transport code } \\
\text { Depletion code }\end{array}$ & WIMS8A & MCNP 4C3 \\
Coupling algorithm & & FISPACT \\
Burn-up steps & 230 & Predictor step \\
$\begin{array}{l}\text { Cross-section } \\
\text { libraries }\end{array}$ & JEF-2.2 based 172-group & JEFF-2.2 based point \\
& cross section library & $\begin{array}{l}\text { energy cross section } \\
\text { library }\end{array}$ \\
\hline
\end{tabular}

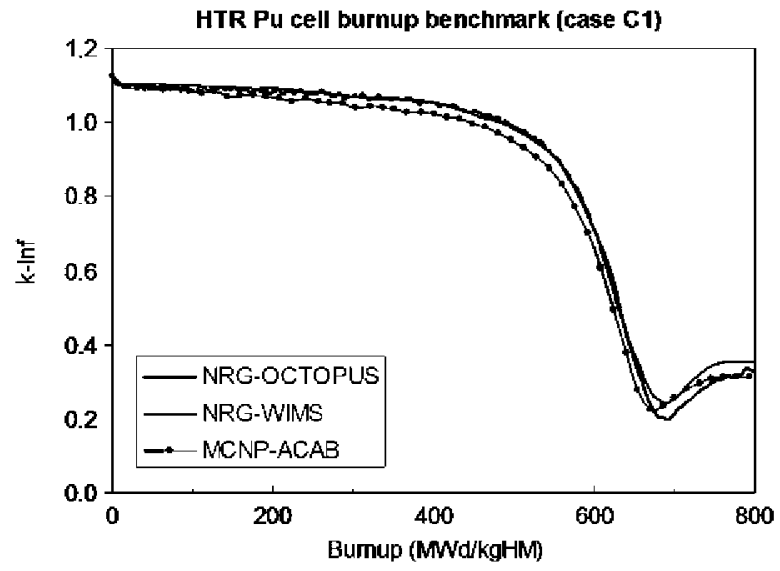

Fig. 5. Infinite multiplication factor as function of burn-up.

burn-up steps taken to reach the requested $800 \mathrm{MWd} /$ $\mathrm{kgHM}$ ) has taken $7.9 \mathrm{~h}$ with 50,000 neutron histories per step.

In Fig. 5, the $k_{\infty}$ is shown as a function of the burn-up. The general shape of the curve predicted by MCNP-ACAB fits with the ones given by NRG: a sharp decrease in reactivity beyond approx. $500 \mathrm{MWd} / \mathrm{kgHM}$, and a slight increase beyond approx. $700 \mathrm{MWd} / \mathrm{kgHM}$. Some numerical details are given in Table 2 .

In Table 3 the density of $\mathrm{Pu}$ isotopes is shown as function of the burn-up. A good agreement is observed between the results of NRG (both WIMS8a and NRG OCTOPUS) and MCNP-ACAB up to a burn-up of approx. $600 \mathrm{MWd} /$ $\mathrm{kgHM}$. At higher burn-up values, the differences in calculated nuclide densities, and consequently in $k$-inf, increase. These discrepancies can be attributed to differences due to the EAF2005 activation library (taken to $300 \mathrm{~K}$ ) as well as differences in the set of nuclides and reactions taken into account in the burn-up calculation. Under usual circumstances (i.e. flux and burn-up levels) these differences will not lead to large differences in results. However, in this particular benchmark both the final burn-up and the flux levels are very high, which greatly amplifies the influence of the differences mentioned above.

For the other actinide and fission product nuclides specified as important in the benchmark, the observed differences between the results are of the same order or magnitude.

\section{Uncertainties in the isotopic inventory for the HTR plutonium cell burn-up exercise: impact of cross-section uncertainties and flux errors}

Let us apply the proposed uncertainty formulations implemented in ACAB to estimate the errors in the actinide inventory for the HTR problem defined above. The actinides under consideration are the ones specified as important in the benchmark.

As first step (Section 5.1), a detailed evaluation of the nuclide density errors is performed considering uncertainties 
Table 2

Infinite multiplication factor along burn-up taking NRG WIMS8A solution as reference

\begin{tabular}{clcc}
\hline Burn-up $(\mathrm{MWd} / \mathrm{kgHM})$ & NRG-WIMS8A $k$-inf & NRG-OCTOPUS $\Delta \rho(\%)$ & MCNP-ACAB $\Delta \rho(\%)$ \\
\hline 0 & 1.1236 & 0.93 & 0.06 \\
100 & 1.0872 & 0.03 & 0.69 \\
400 & 1.0465 & -1.74 & -2.38 \\
600 & 0.71899 & -19.30 & -3.42 \\
800 & 0.35081 & -35.96 \\
\hline
\end{tabular}

For the other solutions, differences in reactivity $\Delta \rho$ with the reference are given.

Table 3

Nuclide densities of $\mathrm{Pu}$ isotopes as function of burn-up, taking NRG-WIMS as reference solution

\begin{tabular}{|c|c|c|c|c|}
\hline Isotopes & Burn-up (MWd/kgHM) & NRG-WIMS $\left(10^{24}\right.$ at. $\left./ \mathrm{cm}^{3}\right)$ & NRG-OCTOPUS $(\%)$ & MCNP-ACAB $(\%)$ \\
\hline \multirow[t]{4}{*}{ Pu-238 } & 100 & $1.07 \mathrm{E}-03$ & 0.05 & 0.14 \\
\hline & 400 & $8.73 E-04$ & -0.58 & 0.07 \\
\hline & 600 & $5.37 \mathrm{E}-04$ & -4.24 & -10.35 \\
\hline & 800 & $4.99 \mathrm{E}-07$ & -33.17 & -25.17 \\
\hline \multirow[t]{4}{*}{ Pu-239 } & 100 & $4.16 \mathrm{E}-03$ & -1.48 & -0.24 \\
\hline & 400 & $6.18 \mathrm{E}-04$ & -7.41 & -1.48 \\
\hline & 600 & $7.39 \mathrm{E}-05$ & -2.10 & -10.31 \\
\hline & 800 & $6.29 \mathrm{E}-08$ & -29.82 & -23.09 \\
\hline \multirow[t]{4}{*}{$\mathrm{Pu}-240$} & 100 & $6.66 \mathrm{E}-03$ & 1.10 & 1.94 \\
\hline & 400 & $2.87 \mathrm{E}-03$ & 1.42 & 7.10 \\
\hline & 600 & $5.77 \mathrm{E}-04$ & -0.71 & -11.64 \\
\hline & 800 & $2.50 \mathrm{E}-06$ & -1.79 & 14.64 \\
\hline \multirow[t]{4}{*}{$\mathrm{Pu}-241$} & 100 & $4.12 \mathrm{E}-03$ & -0.57 & -1.36 \\
\hline & 400 & $2.98 \mathrm{E}-03$ & -1.97 & 0.18 \\
\hline & 600 & $4.37 \mathrm{E}-04$ & -8.37 & -11.91 \\
\hline & 800 & $5.78 \mathrm{E}-07$ & -7.38 & 9.70 \\
\hline \multirow[t]{4}{*}{$\mathrm{Pu}-242$} & 100 & $4.22 \mathrm{E}-03$ & -0.07 & 0.35 \\
\hline & 400 & $4.65 \mathrm{E}-03$ & -0.12 & 2.06 \\
\hline & 600 & $4.95 \mathrm{E}-03$ & -0.55 & 2.80 \\
\hline & 800 & $9.50 \mathrm{E}-04$ & -12.49 & -10.75 \\
\hline
\end{tabular}

For the other solutions the relative difference respect to WIMS is given.

only in the cross-sections, but taking into account the temporal evolution of the neutron flux level and spectrum. As second step, in order to analyze the relative importance of the statistical errors in the calculated flux with respect to the cross-section uncertainties, a simplified problem with constant flux has been defined (Section 5.2). Finally, in Section 5.3 , we justify and validate for this benchmark exercise the assumptions adopted for the Monte Carlo flux error propagation technique.

\subsection{Uncertainties in the isotopic inventory due to cross- section uncertainties}

In the HTR Pu cell burn-up benchmark, uncertainties in the isotopic inventory were only calculated by the NRGOCTOPUS (NRG + FISPACT) scheme. The uncertainties in cross-sections (based upon cross-section uncertainty data from EAF4) were considered as the only error source. The available results are compared in Table 4 with the ones obtained by MCNP-ACAB. In MCNP-ACAB, the Monte Carlo methodology has been used to propagate the crosssection uncertainties (taken from EAF2005) in the isotopic content, following the scheme in Fig. 3. The whole burn-up period has been divided into 50 burn-up steps and MCNP calculations with $50 \mathrm{k}$ neutron histories per step have been performed.

Taking into account the different uncertainty data and different methodologies to propagate uncertainties, the obtained results look satisfactory, being of the same order of magnitude. Differences can also be attributed to the different number of burn-up steps considered by the two systems to reach the requested $800 \mathrm{MWd} / \mathrm{kgHM}$.

\subsection{Uncertainty assessment due to cross-section uncertainties and flux spectrum errors}

For simplicity, only one neutron flux spectrum, corresponding to $400 \mathrm{MWd} / \mathrm{kgHM}$, will be taken for the whole burn-up period. A neutron flux equal to $1.54 \times 10^{15} \mathrm{n} / \mathrm{cm}^{2} \mathrm{~s}$ is considered over the irradiation cycle, being the neutron average energy $\langle E\rangle=0.26 \mathrm{MeV}$.

The cross-section uncertainty data have been taken from the EAF2005/UN library, where uncertainties (relative errors, $\Delta$ ) up to $20 \mathrm{MeV}$ are provided in no more than three 
Table 4

Calculated uncertainties in the $\mathrm{Pu}$ concentrations due to cross-section uncertainties as function of burn-up

\begin{tabular}{|c|c|c|c|}
\hline Isotopes & $\begin{array}{l}\text { Burn-up } \\
(\mathrm{MWd} / \mathrm{kgHM})\end{array}$ & NRG-OCTOPUS $(\%)$ & MCNP-ACAB (\%) \\
\hline $\mathrm{Pu}-238$ & $\begin{array}{l}100 \\
400 \\
600 \\
800\end{array}$ & $\begin{array}{r}1.28 \\
6.72 \\
17.15 \\
50.48\end{array}$ & $\begin{array}{r}0.62 \\
1.90 \\
12.07 \\
26.52\end{array}$ \\
\hline $\mathbf{P u}-239$ & $\begin{array}{l}100 \\
400 \\
600 \\
800\end{array}$ & $\begin{array}{r}5.11 \\
27.04 \\
16.06 \\
46.67\end{array}$ & $\begin{array}{r}3.48 \\
7.92 \\
16.58 \\
23.83\end{array}$ \\
\hline $\mathrm{Pu}-240$ & $\begin{array}{l}100 \\
400 \\
600 \\
800\end{array}$ & $\begin{array}{r}3.77 \\
13.31 \\
25.82 \\
15.39\end{array}$ & $\begin{array}{r}2.88 \\
5.00 \\
12.32 \\
9.89\end{array}$ \\
\hline $\mathrm{Pu}-241$ & $\begin{array}{l}100 \\
400 \\
600 \\
800\end{array}$ & $\begin{array}{r}4.21 \\
9.30 \\
18.30 \\
15.10\end{array}$ & $\begin{array}{r}1.97 \\
4.13 \\
23.78 \\
9.58\end{array}$ \\
\hline $\mathrm{Pu}-242$ & $\begin{array}{l}100 \\
400 \\
600 \\
800\end{array}$ & $\begin{array}{r}2.51 \\
8.95 \\
12.49 \\
66.24\end{array}$ & $\begin{array}{r}1.73 \\
2.59 \\
4.37 \\
24.07\end{array}$ \\
\hline
\end{tabular}

energy-groups and all type of correlations are neglected (the covariance matrices have the off-diagonal values set to zero). We assume the uncertainty values in the library to be three times the experimental relative error, that is, $\Delta_{j, \mathrm{LIBRARY}}=3 \cdot \Delta_{j, \mathrm{EXP}}(j=1$, energy group number $)$, in order to represent a $99.73 \%$ confidence level.

Table 5 illustrates the uncertainties derived from the EAF-2005/UN data file for the $(n, \gamma) \mathrm{Pu}-240$ cross-section reaction. The indicated energy group boundaries change for each reaction and isotope.

Then, if $\hat{\sigma}^{I}$ is best-estimate weighted cross-section in one of the energy groups, the variance of the cross-section in this group due to cross-section uncertainty data is given by:

$\left.\operatorname{var} \sigma^{I}\right|_{\sigma}=\left(\Delta_{\sigma}^{I}\right)^{2} \cdot\left(\hat{\sigma}^{I}\right)^{2}$

The neutron flux spectrum and their relative errors have been obtained in the VITAMINJ group structure from MCNP calculations. Different number of histories have been considered in order to have flux spectrum relative errors of different order of magnitude (see Table 6), that is, different qualities of the transport calculation.

Flux errors are collapsed in the same energy-group structure in which the cross-section uncertainties are given to compute the variance of the cross-section in each group due to flux deviations:

$\left.\operatorname{var} \sigma^{I}\right|_{\phi}=\sum_{g \in I}\left(\sigma^{g}\right)^{2} \operatorname{var} \phi^{g} g=\left(A_{\phi}^{I}\right)^{2} \cdot\left(\hat{\sigma}^{I}\right)^{2}$

For example in Table 7 the data obtained for the $(n, \gamma) \mathrm{Pu}-$ 240 reaction are presented. These uncertainties due to flux errors, with the same structure than uncertainties in Table 5 , are ready to be sampled. Note that for a low number of neutron histories, the flux fluctuations can induce uncertainties in the one-group collapsed cross-sections of the same order of magnitude that the nuclear data errors shown in Table 5.

\subsubsection{Uncertainty assessment by the Monte Carlo method}

Using that uncertainty information, uncertainty assessment of the isotopic inventory has been computed by ACAB along the burn-up cycle by the Monte Carlo methodology. Special emphasis is paid to the Monte Carlo technique, as this approach has a big potential and is relatively new in inventory uncertainty estimations. A log-normal distribution is assumed for the cross-sections in the energy-group structure found in EAF2005/UN, as explained in Section 3.3. A simultaneous random sampling of all the cross-sections involved in the problem is made, obtaining the distributions of the isotopic inventory. A 1000 histories sample size is found appropriate for this application. The obtained actinide uncertainties, for three different neutron history numbers, are in Table 8.

The columns 2-4 refer to the nuclide density errors due to the cross-section uncertainties. Logically, since they are relative errors, they are not very sensitive to the quality of the MCNP transport calculation used to compute the flux spectrum. For most of the nuclides, the concentration uncertainties are higher than $15 \%$, and can reach up to $45 \%$. The fact that the activation cross-section uncertainties in the data files remain high for some isotopes causes those significant uncertainties in the isotopic inventory prediction. Note that the computed results differ from those obtained at $800 \mathrm{MWd} / \mathrm{kgHM}$ in Table 4, where the temporal evolution of the neutron flux level and spectrum were taken into account (the burn-up period was divided into 50 time steps). The differences show the discretization effects of the burn-up period. It is shown that at the unusually high burn-up of this exercise, those discretization effects can be remarkable for some isotopes.

Columns 5-7 show the uncertainties due to the statistical errors. If the MCNP calculation is reliable (flux relative errors lower than $5 \%$, as obtained with $50 \mathrm{k}$ and $500 \mathrm{k}$ neutron histories), the impact of the statistical fluctuations on

Table 5

Cross section relative errors (uncertainties) derived from EAF2005/UN

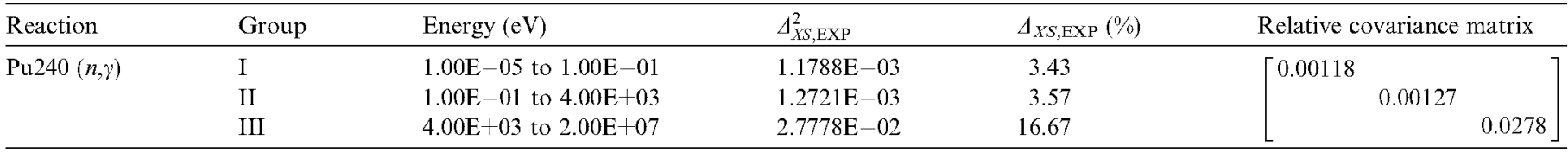


Table 6

Different MCNP calculations to compute the neutron flux spectrum

\begin{tabular}{lll}
\hline Number of histories & $\begin{array}{l}\text { Relative error } \\
(\%) \text { in } k \text {-eff }\end{array}$ & $\begin{array}{l}\text { Order of magnitude } \\
\text { of the relative errors } \\
(\%) \text { in the flux tallies }\end{array}$ \\
\hline $\begin{array}{l}5 \mathrm{k}(50 \text { cycles with } 100 \\
\text { histories/cycle) }\end{array}$ & 1.18 & $\sim 12$ \\
$50 \mathrm{k}(50$ cycles with \\
$\begin{array}{l}1 \mathrm{k} \text { histories/cycle }) \\
500 \mathrm{k}(50 \text { cycles with } \\
10 \mathrm{k} \text { histories/cycle })\end{array}$ & 0.29 & $\sim 5$ \\
\hline
\end{tabular}

Table 7

Cross section relative errors $\left(\Delta_{\phi}=\sqrt{\Delta_{\phi}^{2}}\right)$ due to flux statistical errors

\begin{tabular}{|c|c|c|c|c|}
\hline Reaction & Energy $(\mathrm{eV})$ & $\begin{array}{l}\Delta_{\phi}(\%) \\
(500 \mathrm{k} \\
\text { histories) }\end{array}$ & $\begin{array}{l}\Delta_{\phi}(\%) \\
(50 \mathrm{k} \\
\text { histories) }\end{array}$ & $\begin{array}{l}\Delta_{\phi}(\%) \\
(5 \mathrm{k} \\
\text { histories) }\end{array}$ \\
\hline $\mathrm{Pu}^{240}$ & $1.00 \mathrm{E}-05-1.00 \mathrm{E}-01$ & 0.28 & 0.89 & 2.78 \\
\hline \multirow[t]{2}{*}{$(n, \gamma)$} & $1.00 \mathrm{E}-01-4.00 \mathrm{E}+03$ & 0.29 & 0.95 & 2.97 \\
\hline & $4.00 \mathrm{E}+03-2.00 \mathrm{E}+07$ & 0.076 & 0.24 & 0.76 \\
\hline
\end{tabular}

the density errors is smaller than $5 \%$ in all cases. However, when taking a bad quality of the MCNP calculation (flux relative errors higher than $10 \%$ ), the transmitted errors in the densities can be up to $16 \%$. It is seen that to reduce the error in densities by a factor of 10 , the total number of histories must be increased by a factor of 100 . This tendency is seen, for example, for the density error of $\mathrm{Pu}^{239}$ : the errors are $0.69 \%$ and $6.94 \%$ for $500 \mathrm{k}$ and $5 \mathrm{k}$ histories, respectively.

The total errors in densities due to cross-section and flux uncertainties are shown in columns 8-10. Since the activation cross-section uncertainties are so high, the nuclide errors are sensitive to the flux fluctuations only if the number of neutron histories is low (non-reliable MCNP calculation). In that case, neglecting the effect of flux errors would imply underestimate the density errors up to $10 \%$ $\left(10 \%\right.$ for $\mathrm{Pu}^{238,239}, 6 \%$ for $\left.\mathrm{Pu}^{240,241,242}\right)$. However, if the activation cross-sections were improved (smaller uncertainties), the effect of the statistical flux errors could be significant even with a reliable MCNP calculation.

An analysis of the uncertainty behaviour along burn-up shows that, generally, the concentration uncertainties increase with the irradiation time (see the $\mathrm{Pu}-242$ in Fig. 6). However, exceptions can be found, for example for the Pu-239. The solid lines show the total errors in density (propagating cross-section and flux errors), when the number of histories in the Monte Carlo calculation is 500,000 and 5000. The broken lines show the results when only flux errors have been propagated. Finally, the dot line shows the errors in density when only cross-section errors are propagated. If the number of histories is low, the statistical error has an important effect on the density error.

\subsubsection{Uncertainty evaluation by the sensitivity/uncertainty technique}

The uncertainty estimates have also been computed by the sensitivity/uncertainty technique implemented in $\mathrm{ACAB}$ as described in Section 3.2. For this purpose, the variances of the one-group cross-sections (due to both cross-section uncertainties and statistical flux errors) have been calculated:

$$
\begin{aligned}
\operatorname{var} \sigma^{\mathrm{eff}} & =\left.\operatorname{var} \sigma^{\mathrm{eff}}\right|_{\sigma}+\left.\operatorname{var} \sigma^{\mathrm{eff}}\right|_{\phi} \\
& =\sum_{g=1, \mathrm{II}, \mathrm{III}}\left(\phi^{g}\right)^{2} \Delta_{\sigma}^{g^{2}}\left(\sigma^{g}\right)^{2}+\sum_{g=1, \mathrm{II}, \mathrm{III}} \Delta_{\phi}^{g^{2}}\left(\sigma^{g}\right)^{2}
\end{aligned}
$$

being the relative errors in one-energy group $\Delta_{\text {TOTAL }}^{2}=\frac{\mathrm{var}^{\text {eff }}}{\sigma^{\text {eff }}}=\Delta_{\sigma}^{2}+\Delta_{\phi}^{2}$.

The obtained relative errors for the most important cross-sections are in Table 9. These results allow measuring the relative importance of the two kind of uncertainties for the neutron spectrum characteristic of our problem. Using the large uncertainties available in EAF2005/UN, for a number of neutron histories high enough, the flux error

Table 8

\begin{tabular}{|c|c|c|c|c|c|c|c|c|c|}
\hline \multirow[t]{3}{*}{ Isotope } & \multicolumn{3}{|c|}{ Only due to XS errors } & \multicolumn{3}{|c|}{ Only due to flux errors } & \multicolumn{3}{|c|}{ Total errors } \\
\hline & \multicolumn{3}{|c|}{ Neutron histories } & \multicolumn{3}{|c|}{ Neutron histories } & \multicolumn{3}{|c|}{ Neutron histories } \\
\hline & $500 \mathrm{k}$ & $50 \mathrm{k}$ & $5 \mathrm{k}$ & $500 \mathrm{k}$ & $50 \mathrm{k}$ & $5 \mathrm{k}$ & $500 \mathrm{k}$ & $50 \mathrm{k}$ & $5 \mathrm{k}$ \\
\hline $\mathrm{Pu} 238$ & 19.48 & 19.56 & 19.40 & 0.85 & 2.72 & 8.57 & 19.50 & 19.77 & 21.35 \\
\hline Pu 239 & 15.95 & 16.46 & 16.05 & 0.69 & 2.19 & 6.94 & 15.97 & 16.63 & 17.53 \\
\hline $\mathrm{Pu} 240$ & 20.35 & 19.60 & 19.68 & 0.79 & 2.45 & 7.69 & 20.36 & 19.74 & 21.09 \\
\hline $\mathrm{Pu} 241$ & 19.28 & 19.14 & 18.72 & 0.74 & 2.20 & 6.97 & 19.29 & 19.26 & 19.86 \\
\hline Pu 242 & 46.01 & 47.50 & 46.22 & 1.58 & 5.00 & 16.49 & 46.04 & 47.79 & 48.99 \\
\hline Pu 244 & 7.71 & 7.20 & 7.07 & 0.08 & 0.26 & 0.81 & 7.71 & 7.20 & 7.11 \\
\hline Am 241 & 20.00 & 19.97 & 19.35 & 0.73 & 2.17 & 7.00 & 20.01 & 20.09 & 20.42 \\
\hline Am $242 \mathrm{M}$ & 21.79 & 22.26 & 21.99 & 0.75 & 2.24 & 7.29 & 21.80 & 22.37 & 23.08 \\
\hline Am 243 & 35.46 & 36.39 & 34.86 & 1.18 & 3.71 & 12.09 & 35.47 & 36.58 & 36.64 \\
\hline $\mathrm{Cm} 242$ & 18.32 & 18.89 & 18.78 & 0.23 & 0.66 & 2.25 & 18.33 & 18.90 & 18.90 \\
\hline $\mathrm{Cm} 243$ & 9.72 & 9.47 & 9.62 & 0.28 & 0.78 & 2.74 & 9.72 & 9.50 & 10.04 \\
\hline $\mathrm{Cm} 244$ & 21.41 & 20.79 & 20.65 & 0.79 & 2.43 & 7.56 & 21.42 & 20.92 & 21.86 \\
\hline $\mathrm{Cm} 245$ & 15.38 & 15.14 & 14.88 & 0.57 & 1.75 & 5.55 & 15.39 & 15.23 & 15.78 \\
\hline
\end{tabular}

Relative error (\%) of the final isotopic concentration computed by the Monte Carlo technique

Results are shown at $800 \mathrm{MWd} / \mathrm{kgHM}$. 
contribution to the one-group cross-section is very low and, consequently, it can be neglected. However, if the statistical fluctuations are large (as happens with 5000 histories), the flux errors induce uncertainties in some one-group crosssections of the same order of magnitude that the nuclear data errors, and therefore they should be propagated. This is the case of the $(n, \gamma) \mathrm{Pu}-240$.

Using these effective relative errors and the sensitivity matrix $S$, the uncertainties in nuclide densities due to cross-section or/and statistical flux errors have been computed using Eq. (8). We summarize in Table 10 the obtained uncertainties for the actinides specified in the HTR benchmark when the fluxes have been obtained from MCNP calculations with different number of neutron histories.

The results are very similar to those obtained by the Monte Carlo technique in Table 8 with the corresponding number of histories. The applicability of Monte Carlo and sensitivity/uncertainty approaches has been extensively assessed in a recent study (Sanz et al., 2007) for all the range of burn-up/irradiation times of interest in ADS designs. The same conclusion was drawn there: both methodologies are acceptable to deal with the problem, but using the Monte Carlo one is recommended.

From this study, it can be concluded that:

(i) The two uncertainty methodologies are well implemented in the new updated version of the $\triangle C A B$ code.

(ii) Even at very high burn-ups, such as $800 \mathrm{MWd} / \mathrm{kgHM}$, non-linear effects are not important and the sensitivity method is useful to infer isotopic uncertainties.

(iii) It will be necessary to consider the propagation of the statistical errors for the burn-up calculations if their effect on the one-group collapsed cross-sections is of the same order of magnitude that the effect of the multigroup cross-section uncertainties. This will happen if the MCNP calculation is of a bad quality or if, using a good MCNP calculation, the nuclear data uncertainties in the activation data files were smaller.

\subsection{Verification of the Monte Carlo fux error propagation} methodology

In this section we analyze the purely statistical variation of the neutron flux and their propagation to the isotopic inventory by a extremely demanding parametric study. The purpose is to validate the implemented methodologies in ACAB to propagate the flux errors, that is, to analyze how our results compare with the uncertainties that will be assessed with a parametric technique.

\subsubsection{Analysis of the flux statistical errors}

In order to analyze the purely statistical variation of the neutron flux, and infer an appropriate PDF to be used in our Monte Carlo method, several independent MCNP calculations (identical except by the use of different random number seed) have been run. Three series of $M$ $(M=100)$ Monte Carlo repeated runs have been performed, each one with a different number of neutron histories $(500 \mathrm{k}, 50 \mathrm{k}$ and $5 \mathrm{k})$.

Each series gives 100 samples of the multigroup flux spectrum $\left\{\phi_{1}, \ldots \phi_{175 g}\right\}_{k}, k=1, M$ in the chosen VITAMINJ group structure. The average and standard deviation of the tallied quantities will quantify the uncertainties due to statistical fluctuations:

$\hat{\phi}_{g}=\frac{\sum_{k=1}^{M} \phi_{g k}}{M} \hat{s}^{2}\left(\hat{\phi}_{g}\right)=\frac{\hat{s}^{2}\left(\phi_{g}\right)}{M}=\frac{\sum_{k=1}^{M} \frac{\left(\phi_{g k}-\hat{\phi}_{g}\right)^{2}}{M-1}}{M}$

An analysis of the obtained values shows:

- As the number of histories increases, the $M$ flux values per group tend to a normal distribution (Fig. 7 represents the flux values for the 174 thermal group). This was expected, since in each MCNP calculation, the $\phi_{g}$ given in the output file is the mean value of the fluxes computed for all the histories. Then, as stated by the Central Limit Theorem, as the number of histories approaches infinity, the mean fits a normal distribution.
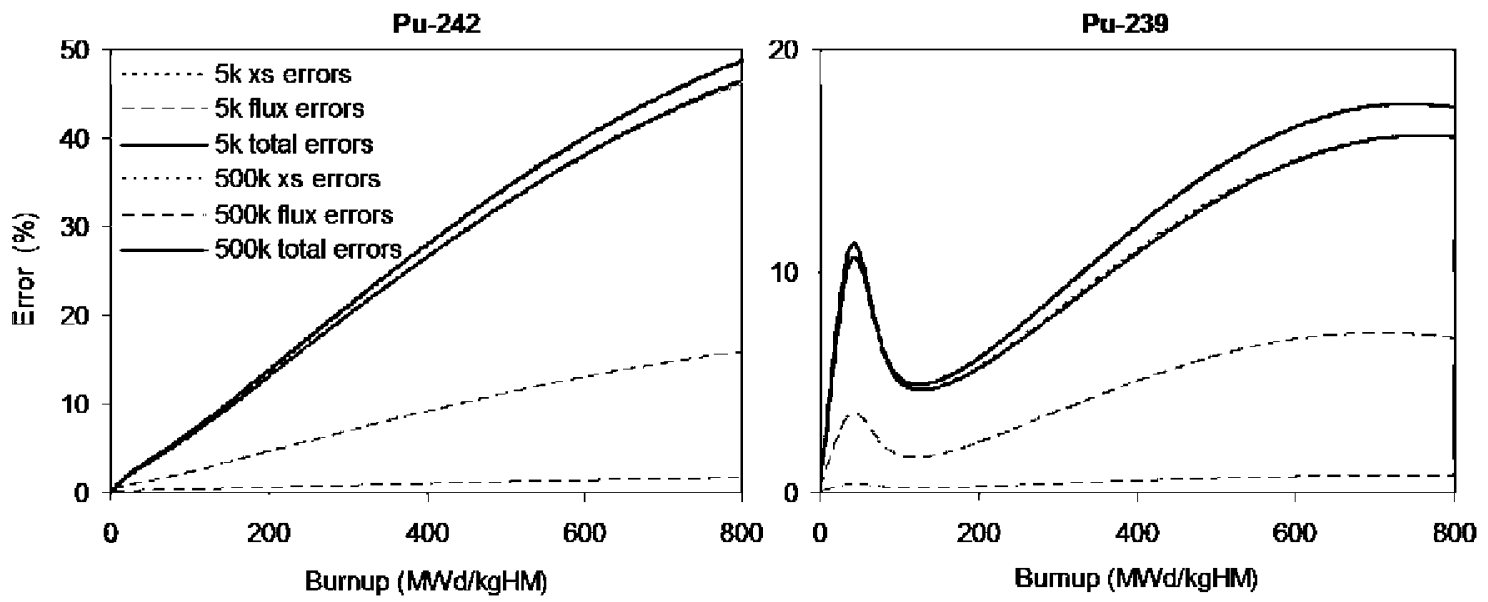

Fig. 6. Comparison between the errors in densities along burn-up for 500k and 5k neutron histories in the MCNP calculation. 
Table 9

One-group cross section relative errors of main reactions $(\%)$ due to cross section uncertainties $\left(\Delta_{\sigma}\right)$, due to flux statistical errors $\left(\Delta_{\phi}\right)$ and due to both $\left(\Delta_{\text {TOTAL }}\right)$

\begin{tabular}{|c|c|c|c|c|c|c|c|c|c|c|}
\hline \multirow[t]{3}{*}{ Reaction } & & \multirow{2}{*}{\multicolumn{3}{|c|}{$\frac{\Delta_{\sigma}}{\text { Neutron histories }}$}} & \multicolumn{3}{|l|}{$\underline{\Delta_{\phi}}$} & \multicolumn{3}{|c|}{$\Delta_{\text {TOTAL }}$} \\
\hline & & & & & \multicolumn{3}{|c|}{ Neutron histories } & \multicolumn{3}{|c|}{ Neutron histories } \\
\hline & & $500 \mathrm{k}$ & $50 \mathrm{k}$ & $5 \mathrm{k}$ & $500 \mathrm{k}$ & $50 \mathrm{k}$ & $5 \mathrm{k}$ & $500 \mathrm{k}$ & $50 \mathrm{k}$ & $5 \mathrm{k}$ \\
\hline \multirow[t]{2}{*}{$\mathrm{Pu}^{238}$} & Fission & 8.29 & 8.30 & 8.31 & 0.11 & 0.35 & 1.10 & 8.29 & 8.30 & 8.38 \\
\hline & Capture & 3.71 & 3.71 & 3.71 & 0.18 & 0.58 & 1.80 & 3.72 & 3.76 & 4.13 \\
\hline \multirow[t]{2}{*}{$\mathrm{Pu}^{239}$} & Fission & 3.33 & 3.33 & 3.33 & 0.17 & 0.54 & 1.76 & 3.33 & 3.37 & 3.76 \\
\hline & Capture & 8.90 & 8.90 & 8.93 & 0.18 & 0.57 & 1.86 & 8.90 & 8.92 & 9.12 \\
\hline \multirow[t]{2}{*}{$\mathrm{Pu}^{240}$} & Fission & 15.44 & 15.47 & 15.36 & 0.10 & 0.30 & 0.95 & 15.44 & 15.47 & 15.39 \\
\hline & Capture & 3.33 & 3.33 & 3.31 & 0.28 & 0.89 & 2.75 & 3.34 & 3.44 & 4.31 \\
\hline \multirow[t]{2}{*}{$\mathrm{Pu}^{241}$} & Fission & 3.30 & 3.30 & 3.30 & 0.15 & 0.48 & 1.54 & 3.31 & 3.34 & 3.64 \\
\hline & Capture & 4.57 & 4.57 & 4.60 & 0.15 & 0.49 & 1.58 & 4.57 & 4.60 & 4.86 \\
\hline \multirow[t]{2}{*}{$\mathrm{Pu}^{242}$} & Fission & 14.88 & 14.87 & 14.87 & 0.10 & 0.32 & 1.03 & 14.88 & 14.88 & 14.91 \\
\hline & Capture & 8.59 & 8.58 & 8.58 & 0.29 & 0.93 & 2.94 & 8.59 & 8.63 & 9.07 \\
\hline \multirow[t]{2}{*}{$\mathrm{Pu}^{244}$} & Fission & 16.37 & 16.37 & 16.37 & 0.11 & 0.36 & 1.16 & 16.37 & 16.37 & 16.41 \\
\hline & Capture & 25.30 & 25.28 & 25.22 & 0.09 & 0.30 & 0.95 & 25.30 & 25.29 & 25.23 \\
\hline
\end{tabular}

Table 10

Relative errors (\%) of the final isotopic concentration computed by the sensitivity technique

\begin{tabular}{|c|c|c|c|c|c|c|c|c|c|}
\hline \multirow[t]{3}{*}{ Isotope } & \multicolumn{3}{|c|}{ Only due to XS errors } & \multicolumn{3}{|c|}{ Only due to flux errors } & \multicolumn{3}{|c|}{ Total errors } \\
\hline & \multicolumn{3}{|c|}{ Neutron histories } & \multicolumn{3}{|c|}{ Neutron histories } & \multicolumn{3}{|c|}{ Neutron histories } \\
\hline & $500 \mathrm{k}$ & $50 \mathrm{k}$ & $5 \mathrm{k}$ & $500 \mathrm{k}$ & $50 \mathrm{k}$ & $5 \mathrm{k}$ & $500 \mathrm{k}$ & $50 \mathrm{k}$ & $5 \mathrm{k}$ \\
\hline Pu 238 & 19.13 & 19.14 & 19.03 & 0.88 & 2.78 & 8.62 & 19.15 & 19.34 & 20.90 \\
\hline Pu 239 & 16.03 & 16.04 & 15.95 & 0.71 & 2.25 & 6.95 & 16.05 & 16.20 & 17.40 \\
\hline Pu 240 & 20.82 & 20.75 & 20.53 & 0.78 & 2.47 & 7.80 & 20.83 & 20.90 & 21.96 \\
\hline $\mathrm{Pu} 241$ & 20.09 & 20.02 & 19.79 & 0.70 & 2.24 & 7.07 & 20.10 & 20.14 & 21.02 \\
\hline Pu 242 & 46.45 & 46.35 & 46.08 & 1.58 & 5.00 & 15.79 & 46.47 & 46.62 & 48.71 \\
\hline Pu 244 & 7.02 & 7.01 & 6.95 & 0.08 & 0.26 & 0.84 & 7.02 & 7.02 & 7.00 \\
\hline Am 241 & 20.70 & 20.63 & 20.48 & 0.70 & 2.22 & 7.04 & 20.71 & 20.75 & 21.66 \\
\hline Am $242 \mathrm{M}$ & 22.45 & 22.44 & 22.25 & 0.71 & 2.27 & 7.20 & 22.46 & 22.56 & 23.38 \\
\hline Am 243 & 36.25 & 36.23 & 36.16 & 1.17 & 3.71 & 11.75 & 36.26 & 36.42 & 38.02 \\
\hline $\mathrm{Cm} 242$ & 18.32 & 18.32 & 18.22 & 0.22 & 0.70 & 2.25 & 18.32 & 18.33 & 18.36 \\
\hline Cm 243 & 9.39 & 9.38 & 9.43 & 0.24 & 0.76 & 2.54 & 9.39 & 9.41 & 9.76 \\
\hline $\mathrm{Cm} 244$ & 22.03 & 21.96 & 21.72 & 0.76 & 2.42 & 7.64 & 22.04 & 22.09 & 23.03 \\
\hline $\mathrm{Cm} 245$ & 15.86 & 15.80 & 15.54 & 0.55 & 1.75 & 5.48 & 15.87 & 15.89 & 16.48 \\
\hline
\end{tabular}

Results are shown at $800 \mathrm{MWd} / \mathrm{kgHM}$.

- As expected, the standard deviations in the flux spectrum due to statistical fluctuations are smaller as the number of histories in the statistical sample increase (Fig. 7). The statistical deviation in the results decreases

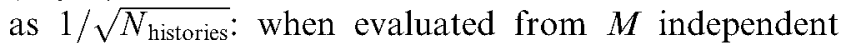
5000-histories runs, $\hat{s}\left(\hat{\phi}_{g}\right)=1.1 \mathrm{E}-3$; increasing the total number of histories by a factor of 100 (500k-histories runs), $\hat{s}\left(\hat{\phi}_{g}\right)=9.3 \mathrm{E}-5$, that is, the standard deviation is reduced about 10 times. Running an infinite number of histories or repeated calculations would reduce the statistical deviations to zero.

- No correlations between energy groups.

The question is: are the statistical deviations in the flux calculated by the above method of the same order of magnitude that the relative errors obtained in a single MCNP calculation?
The estimated relative error given by a single MCNP calculation with $N$ neutron histories is the estimated standard deviation of the mean divided by the estimated mean $\hat{s}\left(\hat{\phi}_{g}\right) / \hat{\phi}_{g}$. It is of the same order of magnitude than the purely statistical error calculated by Eq. (9) from a series of $M$ repeated calculations with the same total number of histories $\left(M * N^{\prime}\right.$, where $N^{\prime}$ is the number of histories in each single calculation). The relative error in a single MCNP calculation with $500 \mathrm{k}$ histories is $0.21 \%$ for the 174 -group flux, similar to the purely statistical error of $0.26 \%$ found when analyzing 100 independent samples with $5 \mathrm{k}$ histories/sample.

In conclusion, it can be assumed that the flux spectrum fits a normal distribution $\left(\phi^{g} / \hat{\phi}^{g}\right) \rightarrow N\left(1, \hat{s}\left(\hat{\phi}_{g}\right)\right.$ $\left./ \hat{\phi}^{g}\right)$, where the relative error is the statistical uncertainty in the flux directly taken from a single MCNP calculation. 

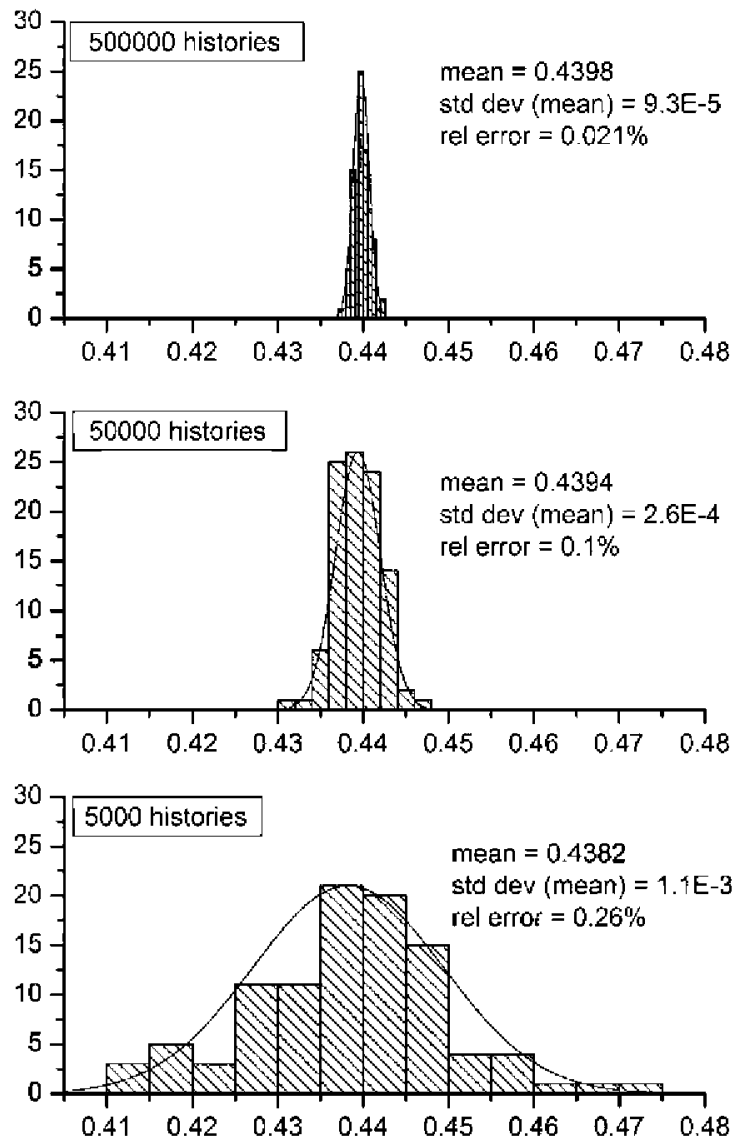

Fig. 7. Probability density functions of the calculated flux for the 174 thermal energy group. The flux distributions have been calculated from 100 samples, each one having the number of neutron histories indicated in the figure.

\subsubsection{Statistical analysis to estimate the flux errors effect on the isotopic inventory}

For each series of $M$ MCNP repeated calculations, we have obtained $M$ samples of the nuclide concentrations $\left\{N_{k}\right\}, k=1, M$, computed by ACAB. For any isotope $N_{i}$, the mean and standard deviation of the mean can be computed as:

$\widehat{N}_{i}=\frac{\sum_{k=1}^{M} N_{i k}}{M} \quad \hat{s}^{2}\left(\widehat{N}_{i}\right)=\frac{\sum_{k=1}^{M} \frac{\left(N_{i k}-\widehat{N}_{i}\right)^{2}}{M-1}}{M}$

The calculated standard deviations quantify the uncertainties in the inventory due to statistical fluctuations from the individual Monte Carlo runs. The obtained results for all the actinides of interest in the HTR-benchmark are shown in Table 11. For $\mathrm{Pu}-239$, values are represented in Fig. 8.

It is verified that the statistical uncertainties in the nuclide concentration decrease as $1 / \sqrt{N_{\text {histories }}}$, that is, $\Delta_{\text {statistical }} \propto 1 / \sqrt{N_{\text {histories }}}$. For Pu-242, the error running 100 separate calculations with $5 \mathrm{k}$ histories is $1.58 \%$, ten times higher than the computed error from calculations with $500 \mathrm{k}$ histories, $0.15 \%$. The Central Limit Theorem states that as $M$ approaches infinity, there is a $99 \%$ chance that the true concentrations will be in the range $\widehat{N}_{i}\left(1 \pm 3 \hat{s}_{i} / \widehat{N}_{i}\right)$.
Table 11

Relative error (\%) of the final isotopic concentration due to the purely flux statistical errors

\begin{tabular}{llll}
\hline Isotope & \multicolumn{3}{l}{ Total neutron histories } \\
\cline { 2 - 4 } & $\mathrm{M}^{*} 500 \mathrm{k}$ & $\mathrm{M}^{*} 50 \mathrm{k}$ & $\mathrm{M}^{*} 5 \mathrm{k}$ \\
\hline Pu 238 & 0.11 & 0.28 & 1.00 \\
Pu 239 & 0.10 & 0.27 & 0.99 \\
Pu 240 & 0.08 & 0.23 & 0.76 \\
Pu 241 & 0.07 & 0.21 & 0.63 \\
Pu 242 & 0.15 & 0.48 & 1.58 \\
Pu 244 & 0.01 & 0.03 & 0.09 \\
Am 241 & 0.07 & 0.22 & 0.65 \\
Am 242M & 0.07 & 0.22 & 0.68 \\
Am 243 & 0.11 & 0.37 & 1.22 \\
Cm 242 & 0.03 & 0.08 & 0.28 \\
Cm 243 & 0.04 & 0.13 & 0.44 \\
Cm 244 & 0.08 & 0.22 & 0.70 \\
Cm 245 & 0.05 & 0.16 & 0.51 \\
\hline
\end{tabular}

Results are shown at $800 \mathrm{MWd} / \mathrm{kgHM}$.

It can be concluded that, for this benchmark, the statistical effect on the nuclide density is smaller than $1.5 \%$ if the number of total histories is higher than 500k. Applying the same scaling behaviour, a reduction in the neutron histories will induce larger errors on the nuclide concentrations.
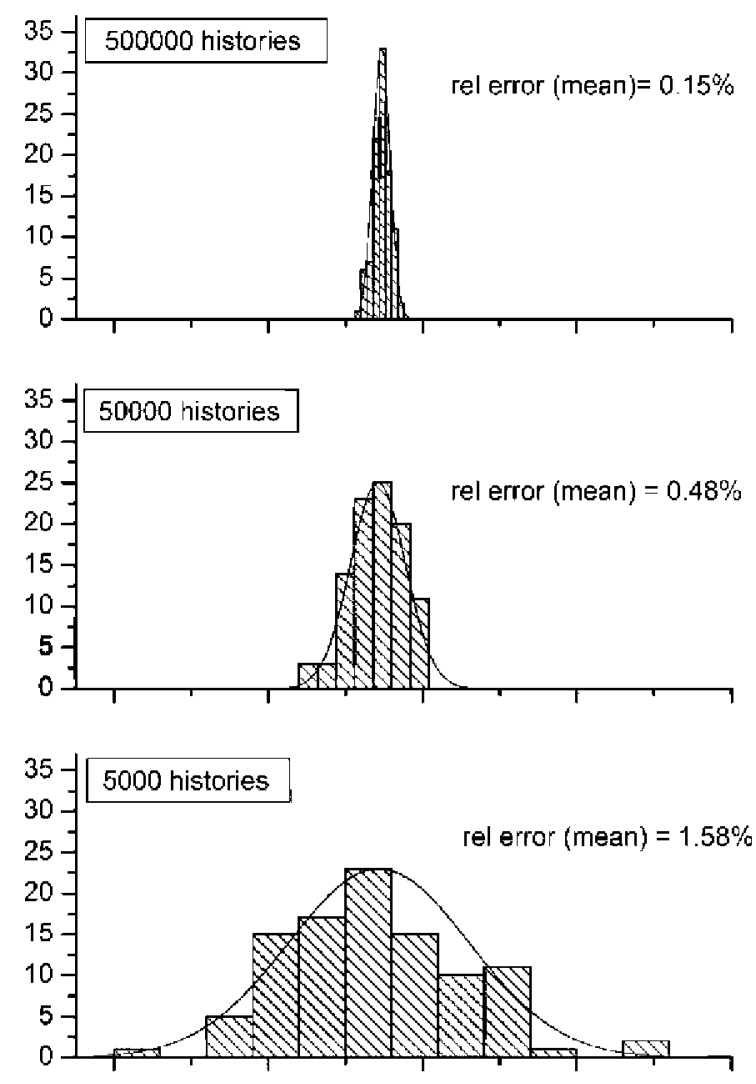

Fig. 8. Pu-242 concentration distribution computed with ACAB. The concentration distributions have been calculated from 100 samples, each one taken a flux spectrum obtained running a MCNP calculation with a number of neutron histories indicated in the figure. 
The question is: can the errors in the concentrations in Table 11 calculated by the above method be predicted from a single MCNP calculation? Let us compare with the concentrations computed by our methodology in Table 8 .

Comparing Tables 8 and 11, we observe that the purely statistical errors in Table 11 obtained from $M$ independent MCNP calculations of $N$ neutron histories are very similar to the errors computed from the flux data obtained in a single MCNP calculation with $\mathrm{M}^{*} N$ neutron histories, that is, if summing up the same number of total histories. For example, the relative error in the $\mathrm{Pu}-242$ concentration in Table 8 obtained with $500 \mathrm{k}$ histories is $1.58 \%$; equal to the purely statistical error found in Table 11 from 100 samples of $5 \mathrm{k}$ histories/sample. Next conclusions are then obtained:

- First, the implemented Monte Carlo method in ACAB is demonstrated to be adequate to propagate the statistical uncertainties in the flux on the isotopic inventory. It guarantees that there is a $99 \%$ chance that the true result will be in the range [estimated concentration $(1 \pm$ relative error)].

- Second, as it was previously obtained, the effect of the flux errors on the isotopic inventory can be neglected if the number of neutron histories is high enough to guarantee a small relative error in the flux spectrum. Otherwise, the flux errors have to be propagated in isotopic inventory predictions.

\section{Conclusions}

In summary, a new automated tool called MCNPACAB, that links the Monte Carlo transport code MCNP-4C with our inventory code ACAB is presented. It enables to estimate the impact of neutron cross-section uncertainties as well as neutron flux statistical errors on the inventory in transport-burn-up combined problems, by using either a sensitivity/uncertainty or a Monte Carlo propagation technique.

The full system has been successfully applied to a HTR benchmark and it has been demonstrated to be reliable to compute accurate high burn-up isotopic inventory with uncertainty estimates. It is concluded that both Monte Carlo and sensitivity/uncertainty methodologies are acceptable to deal with this problem.

The computed nuclide errors due to the cross-section uncertainties available at present in the data files are very large. In regards to the flux error impact, the results show that when the flux spectrum is obtained from a reliable stochastic transport calculation, the influence of the flux statistical deviations on the isotopic inventory is negligible (even at very high burn-up) compared to the effect of the cross-section uncertainties. If the stochastic transport calculation is not reliable (flux relative errors higher than $10 \%$ ), the impact of the statistical errors is not negligible on some isotopes, even if the contribution is mainly due to the cross-section errors.

In consequence, to evaluate if the impact of the flux statistical errors is negligible or not, we recommend to compute the errors of the one-group collapsed cross-sections. A comparison between the contribution of the flux statistical deviations and the cross-section uncertainties will allow to estimate if it is necessary to propagate the statistical errors.

\section{References}

Briesmeister, J.F. (Ed.), 2000. MCNPтM - A General Monte Carlo $\mathrm{N}$ Particle Transport Code, Version 4C. LA-13709-M, Los Alamos National Laboratory.

Cabellos, O., Sanz, J., Rodríguez, A., González, E., Embid, M., Álvarez, F., Reyes, S., 2005. Sensitivity and Uncertainty Analysis to Burn-up Estimates on $\mathrm{ADS}$ using the $\mathrm{ACAB}$ Code. American Institute of Physics (AIP) Conf. Proceedings, vol. 769, 1576.

Cabellos, O. et al., 2006. Effect of activation cross section uncertainties on the radiological assessment of the MFE/DEMO first wall. Fusion Engineering and Design 81 (8-14), 1561-1565.

Forrest, R.A., Sublet, J-Ch., 2001. FISPACT-2001: User Manual. UKAEA FUS 450, EURATOM/UKAEA.

Garcia-Herranz, N., Cabellos, O., Sanz, J., 2005. Applicability of the MCNP-ACAB system to inventory prediction in high burn-up fuels: sensitivity/uncertainty estimates. In: Proc. Int. Conf. on Mathematics and Computation, M\&C2005, Avignon, France.

Ivanov, E., 2005. Error propagation in Monte-Carlo burn-up calculations. In: Proc. Int. Conf. on Mathematics and Computation, M\&C2005, Avignon, France.

Kuijper, J.C., et al., 2004. HTR-N Plutonium Cell Burn-up Benchmark: Definition, Results \& Intercomparison. PHYSOR-2004, Chicago, Illinois.

Kuijper, J.C., Oppe, J., Klein Meulekamp, R., Koning, H., 2005. Propagation of cross section uncertainties in combined Monte Carlo neutronics and burn-up calculations. In: Proc. Int. Conf. on Mathematics and Computation, M\& C2005, Avignon, France.

NEA Data Bank Computer Programs, 2002. ORIGEN2.2; Isotope Generation and Depletion Code, Matrix Exponential Method. Document CCC-0371, Oak Ridge National Laboratory.

Oppe, J., Kuijper, J.C., 2004. OCTOPUS_TNG Reference Guide. NRG report 20748/04.54103, NRG, Petten, The Netherlands.

Poston, D.I., Trellue, H.R., 2002. MONTEBURNS Version 1.0 User's Manual Version 2.0. LA-UR-99-4999, Los Alamos National Laboratory.

Sanz, J., 2000. ACAB Activation Code for Fusion Applications: User's manual V5.0. UCRL-MA-143238, Lawrence Livermore National Laboratory.

Sanz, J., Falquina, R., Rodríguez, A., Cabellos, O., Latkowski, J.F., Reyes, S., 2003. Monte carlo uncertainty analysis of pulsed activation in the NIF gunite shielding. Fusion Science and Technology 43, 473477.

Sanz, J., Cabellos, O., Reyes, S., 2004. Effect of activation cross-section uncertainties in selecting steels for the HYLIFE-II chamber to successful waste management. Fusion Engineering and Design, 11571161. 
Sanz, J., et al., 2006. Interim Report on the description of selected nuclear data sensitivity methodologies for the fuel cycle and the repository parameters, Deliverable D5.1, Domain DM5 NUDATRA, EUROTRANS Project.

Sanz, J., et al., 2007. Description of selected methodologies for propagation of cross-section uncertainties to fuel cycle and repository parameters: Application to the prediction of actinide inventory. Deliverable D5.10, Domain DM5 NUDATRA, EUROTRANS Project.

Takeda, T., Hirokawa, N., Noda, T., 1999. Estimation of error propagation in Monte-Carlo burn-up calculations. Journal of Nuclear Science and Technology 36, 738-745.
Tippayakul, C., Ivanov, K., Misu, S., 2006. Improvements of MCOR: a Monte Carlo Depletion Code System for Fuel Assembly Reference Calculations. PHYSOR-2006, Vancouver, BC, Canada.

Tohjoh, M., Endo, T., Watanabe, M., Yamamoto, A., 2006. Effect of error propagation of nuclide number densities on Monte Carlo burn-up calculations. Annals of Nuclear Energy 33 (17-18), 1424 1436.

Xu, Z., Hejzlar, P., Driscoll, M.J., Kazimi, M.S., 2002. An improved MCNP-ORIGEN depletion program (MCODE) and its verification for high burn-up applications. In: Proc. of the Int. Conf. PHYSOR 2002, Seoul, Korea. 